TRANSACTIONS OF THE

AMERICAN MATHEMATICAL SOCIETY

Volume 284. Number I. July 1984

\title{
POSITIVE-DEFINITENESS AND ITS APPLICATIONS TO INTERPOLATION PROBLEMS FOR HOLOMORPHIC FUNCTIONS
}

BY

FRANK BEATROUS, JR. AND JACOB BURBEA

\begin{abstract}
Holomorphic interpolation problems of the Pick-Nevanlinna and Loewner types as well as abstract interpolation theorems on functional Hilbert spaces are considered. Various characterizations are presented for restrictions of bounded holomorphic functions. In addition, certain norm estimates for restrictions and extensions of holomorphic functions are obtained.
\end{abstract}

1. Introduction. In this paper we study positive-definiteness of certain kernels by exploiting a natural identification of these kernels with operators on an appropriate Hilbert space. This identification allows us to establish certain interpolation theorems of an abstract character. As an application of this general study we also consider some interpolation problems for various classes of holomorphic functions. Thus we wish to characterize the restriction of a class of holomorphic functions on a domain $D$ in $\mathbf{C}^{n}$ to some small subset $E$ of $\bar{D}$. Our results fall into two categories: interpolation theorems for Hilbert spaces of holomorphic functions, and interpolation theorems for bounded holomorphic functions.

The first type of problem has been studied previously by FitzGerald [14], and the present work is partially motivated by his paper (see also FitzGerald and Horn [16] and Donoghue [12]). Our methods allow us to extend the results of FitzGerald considerably (see Theorems 3.1 and 3.4).

The problem of interpolating bounded holomorphic functions goes back to Pick [24] and Nevanlinna [23]. Their result characterizes restrictions of bounded holomorphic functions on the unit disk $\Delta$ by a quadratic inequality involving the Szegö kernel $S_{\Delta}(z, \zeta)=[2 \pi(1-z \bar{\zeta})]^{-1}$ of $\Delta$. Here we prove an analogous result where $\Delta$ is replaced by a domain $D$ in $\mathbf{C}^{n}$ and $S_{\Delta}(z, \zeta)$ is replaced by an arbitrary positive-definite sesqui-holomorphic kernel on $D$ (see Theorem 3.5).

In spite of the different characters of the above two types of problems, it has been possible to link them here via the notion of multiplication-operator (see Proposition 3.6). This link was instrumental in unifying this paper and enabled us to derive various other interpolation theorems. In particular, it enabled us to establish some interpolation theorems for bounded holomorphic functions from a subset of the boundary (see Theorems 4.3 and 4.6). These theorems extend a previous result of Beatrous [2] which was proved using different methods and with more restrictive assumptions.

Received by the editors February 16, 1983

1980 Mathematics Subject Classification. Primary 30C40, 30C55, 32H10, 32A10, 32A35, 46E20.

Key words and phrases. Positive-definiteness, functional Hilbert spaces, holomorphic interpolation. 
The present results admit a vectorial generalization on a theme set forth in Burbea $[4,6,8]$ and Burbea and Masani $[9,10]$. We shall not pursue these points here, but leave them for another occasion.

The paper is organized as follows: In $\$ 2$ we derive some abstract interpolation theorems for functional Hilbert spaces. Here, a fundamental tool is Aronszajn's general theory of reproducing kernels [1]. In $\$ 3$ we specialize to spaces of holomorphic functions, and prove the theorems mentioned above. We also give applications to interpolation problems for certain Hardy spaces and Bergman spaces on the ball and polydisk, as well as for certain spaces of entire functions. In $\$ 4$ we prove the boundary analogues of the theorems mentioned above.

2. Abstract interpolation theorems. We begin with some definitions. For any nonempty set $X$, a kernel on $X$ is a complex-valued function on $X \times X$. A kernel $K$ on $X$ is hermitian or positive-definite on $X$ if for any finite sequence $x_{1}, \ldots, x_{n}$ of points in $X$, the matrix [ $\left.K\left(x_{i}, x_{j}\right)\right]$ is hermitian or positive-definite, respectively.

A Hilbert space $\mathscr{H}$, each of whose members is a function on $X$ is a functional Hilbert space on $X$ if for each $x \in X$, the functional $f \rightarrow f(x)$ is continuous from $\mathscr{H}$ to $\mathrm{C}$. In this case there is, for each $x \in X$, a unique function $k_{x} \in \mathscr{H}$ with the property that $f(x)=\left\langle f, k_{x}\right\rangle$ for every $f \in \mathscr{H}$. Moreover, the kernel $K(x, y)=$ $k_{y}(x)=\left\langle k_{y}, k_{x}\right\rangle$ is positive-definite on $X$. The kernel $K$ is called the reproducing kernel of the functional Hilbert space $\mathscr{H}$.

Many interesting examples of functional Hilbert spaces arise as spaces of holomorphic functions. For example, the space $A^{2}(D)$ of square integrable holomorphic functions on a domain $D$ in $\mathbf{C}^{n}$ forms a functional Hilbert space with the usual $L^{2}$-inner product. Similarly, if $D$ is a domain with a $C^{2}$ boundary (or a cartesian product of such domains) then the Hardy space $H^{2}(D)$ consisting of all holomorphic functions on $D$ with square integrable boundary values forms a functional Hilbert space on $D$. The reproducing kernels associated with $A^{2}(D)$ and $H^{2}(D)$ are the classical Bergman and Szegö kernel functions.

We will need the following fundamental result of Aronszajn [1].

2.1. TheOREM. Let $K$ be a positive-definite kernel on $X$. There exists a unique - functional Hilbert space $\mathscr{H}$ on $X$ with $K$ as its reproducing kernel. Moreover, the vector space $\mathscr{H}_{0}$ consisting of functions of the form $\sum \alpha_{i} K\left(\cdot, x_{i}\right)$, where $\left\{x_{i}\right\}$ and $\left\{\alpha_{i}\right\}$ are finite sequences in $X$ and $\mathbf{C}$ respectively, is a dense subspace of $\mathscr{H}$. The inner product in $\mathscr{H}_{0}$ is given by

$$
\left\langle\sum \alpha_{i} K\left(\cdot, x_{i}\right), \sum \beta_{j} K\left(\cdot, y_{j}\right)\right\rangle=\sum \alpha_{i} \bar{\beta}_{j} K\left(y_{j}, x_{i}\right) .
$$

Thus there is a natural one-to-one correspondence between positive-definite kernels on $X$ and functional Hilbert spaces on $X$.

For the remainder of this section, we fix a nonempty set $X$ and a positive-definite kernel $K$ on $X$, and we let $\mathscr{H}=\mathscr{H}(X ; K)$ denote the associated functional Hilbert space on $X$.

The following theorem is an abstract version of a result of FitzGerald [14] (see also FitzGerald and Horn [16]) and it should also be compared with a corresponding abstract result of Aronszajn [1] (see also Burbea [4, 6] and Donoghue [12]). 
2.2 THEOREM. A function $f$ on $X$ lies in $\mathscr{H}$ if and only if there is a positive constant $c$ such that the kernel $c^{2} K(x, y)-f(x) \overline{f(y)}$ is positive-definite on $X$. In this case, $\|f\|$ is the infimum of all such $c$.

Proof. First assume that the kernel $K_{f}(x, y)=c^{2} K(x, y)-f(x) \overline{f(y)}$ is positive-definite on $X$. We will show that $f$ is in $\mathscr{H}$. If $f=0$ there is nothing to prove, so we may assume that $f\left(x_{0}\right) \neq 0$ for some point $x_{0} \in X$. Writing $K(x, y)=$ $c^{-2}\left[K_{f}(x, y)+f(x) \overline{f(y)}\right]$, we see that $K$ is the sum of two positive-definite kernels $K_{f}$ and $f(x) \overline{f(y)}$ on $X$. It follows from the general theory of reproducing kernels (see Aronszajn [1]) that $\mathscr{H}$ is the pointwise sum of the functional Hilbert spaces associated with the kernels $K_{f}$ and $f(x) \overline{f(y)}$. In particular, the function $h=$ $K_{f}\left(\cdot, x_{0}\right)+2 \overline{f\left(x_{0}\right)} f$ is in $\mathscr{H}$. Thus $f=\left\{\overline{f\left(x_{0}\right)}\right\}^{-1}\left[h-c^{2} K\left(\cdot, x_{0}\right)\right]$, which is evidently in $\mathscr{H}$.

For the converse, assume that $f$ is in $\mathscr{H}$ and consider the kernel $K_{f}(x, y)=$ $c^{2} K(x, y)-f(x) \overline{f(y)}$, where $c$ is some positive number. For any finite sequences $\left\{x_{i}\right\}$ in $X$ and $\left\{\alpha_{i}\right\}$ in $\mathbf{C}$ we have

$$
\begin{aligned}
\sum \alpha_{i} \bar{\alpha}_{j} K_{f}\left(x_{i}, x_{j}\right) & =c^{2} \sum \alpha_{i} \bar{\alpha}_{j} K\left(x_{i}, x_{j}\right)-\left|\sum \alpha_{i} f\left(x_{i}\right)\right|^{2} \\
& =c^{2} \sum \alpha_{i} \bar{\alpha}_{j} K\left(x_{i}, x_{j}\right)-\left|\sum \alpha_{i}\left\langle f, K\left(\cdot, x_{i}\right)\right\rangle\right|^{2} \\
& =c^{2}\left\|\sum \bar{\alpha}_{i} K\left(\cdot, x_{i}\right)\right\|^{2}-\left|\left\langle f, \sum \bar{\alpha}_{i} K\left(\cdot, x_{i}\right)\right\rangle\right|^{2} .
\end{aligned}
$$

Thus it follows from the Cauchy-Schwarz inequality that $K_{f}$ is positive-definite on $X$ if $c \geqslant\|f\|$.

Note also that, since functions of the form $\sum \bar{\alpha}_{i} K\left(\cdot, x_{i}\right)$ are dense in $\mathscr{H}$, the positive-definiteness of $K_{f}$ implies that $c^{2}\|g\|^{2} \geqslant|\langle f, g\rangle|^{2}$ for all $g \in \mathscr{H}$. In particular, taking $g=f$, it follows that $c \geqslant\|f\|$. This concludes the proof.

We will say that a kernel $L$ on $X$ is subordinate to $K$ if there is a nonnegative constant $c$ such that for all finite sequences $\left\{x_{i}\right\}$ in $X$ and $\left\{\alpha_{i}\right\},\left\{\beta_{i}\right\}$ in $\mathbf{C}$ we have

$$
\left|\sum \alpha_{i} \bar{\beta}_{j} L\left(x_{i}, x_{j}\right)\right|^{2} \leqslant c^{2}\left(\sum \alpha_{i} \bar{\alpha}_{j} K\left(x_{i}, x_{j}\right)\right)\left(\sum \beta_{i} \bar{\beta}_{j} K\left(x_{i}, x_{j}\right)\right) .
$$

If we define $\|L\|$ to be the infimum of all nonnegative constants $c$ for which the above inequality holds, then the set $\mathscr{S}(K)$ of kernels subordinate to $K$ becomes a Banach space under pointwise operations.

2.3. LemMA. If $L$ is a kernel on $X$ which is subordinate to $K$, i.e. if $L \in \mathscr{S}(K)$, then for any $x_{0} \in X$ the functions $L\left(\cdot, x_{0}\right)$ and $\overline{L\left(x_{0}, \cdot\right)}$ are in $\mathscr{H}$.

Proof. Condition (2.1) implies in particular that

$$
\left|\sum \alpha_{i} L\left(x_{i}, x_{0}\right)\right|^{2} \leqslant c_{0}^{2} \sum \alpha_{i} \bar{\alpha}_{j} K\left(x_{i}, x_{j}\right)
$$

where $c_{0} \equiv c \sqrt{K\left(x_{0}, x_{0}\right)}$. Thus the kernel $c_{0}^{2} K(x, y)-L\left(x, x_{0}\right) \overline{L\left(y, x_{0}\right)}$ is positive-definite on $X$, so by Theorem $2.2, L\left(\cdot, x_{0}\right)$ is in $\mathscr{H}$. To see that $\overline{L\left(x_{0}, \cdot\right)}$ lies in $\mathscr{H}$, we observe that $L^{*}(x, y)=\overline{L(y, x)}$ is also subordinate to $K$ and apply the above argument to $L^{*}$. 
We may also consider the space $\mathscr{S}_{1}(K)$ of all kernels $L$ on $X$ such that there is a nonnegative constant $c$ with the property that

$$
\left|\sum \alpha_{i} \bar{\alpha}_{j} L\left(x_{i}, x_{j}\right)\right| \leqslant c \sum \alpha_{i} \bar{\alpha}_{j} K\left(x_{i}, x_{j}\right)
$$

for all finite sequences $\left\{x_{i}\right\}$ in $X$ and $\left\{\alpha_{i}\right\}$ in C. Again, $\|L\|_{1}$ is defined as the infimum of all nonnegative constants $c$ for which (2.2) holds. Note that $\|\cdot\|_{1}$ is a seminorm. Clearly, $\mathscr{S}(K) \subset \mathscr{S}_{1}(K)$ and $\|L\|_{1} \leqslant\|L\|$ for every $L \in \mathscr{S}(K)$. For any kernel $L$ on $X$, we define its adjoint $L^{*}$ by $L^{*}(x, y)=\overline{L(y, x)}$. It follows that $L^{*} \in \mathscr{S}(K)$ or $L^{*} \in \mathscr{S}_{1}(K)$ if and only if $L \in \mathscr{S}(K)$ or $L \in \mathscr{S}_{1}(K)$, in which case $\left\|L^{*}\right\|=\|L\|$ or $\left\|L^{*}\right\|_{1}=\|L\|_{1}$, respectively. The proof of the next lemma is quite standard and therefore omitted.

2.4. Lemma. If $L \in \mathscr{S}_{1}(K)$ then its hermitian part $\left(L+L^{*}\right) / 2$ is in $\mathscr{S}(K)$ and $\left\|L+L^{*}\right\|=2\|L\|_{1}$. In particular, for $L \in \mathscr{S}(K),\|L\|=\|L\|_{1}$ if and only if $L$ is hermitian on $X$ (i.e. if $L$ is selfadjoint, $\left.L^{*}=L\right)$.

A fundamental theme of this paper will be the reduction of problems concerning kernels to analogous questions concerning operators on an appropriate Hilbert space. The reduction is facilitated by the following result (compare Aronszajn [1]). Here $\mathscr{B}(\mathscr{H})$ denotes the Banach algebra of continuous linear operators from $\mathscr{H}$ to itself.

2.5. TheOREM. For any operator $T \in \mathscr{B}(\mathscr{H})$ there is a unique kernel $L_{T} \in \mathscr{S}(K)$ which represents $T$ in the sense that $\{T f\}(y)=\left\langle f, L_{T}(\cdot, y)\right\rangle$ for every $f \in \mathscr{H}$ and every $y \in X$. This kernel is given by $L_{T}(x, y)=\{T K(\cdot, x)\}(y)$ and $L_{T^{*}}(x, y)$ $=\overline{L_{T}(y, x)}$ for $x, y \in X$. Moreover, the mapping $T \mapsto L_{T}$ is a conjugate linear isometry of $\mathscr{B}(\mathscr{H})$ onto $\mathscr{S}(K)$ with the property that $L_{T}$ is an hermitian or a positive-definite kernel on $X$ if and only if $T$ is a selfadjoint or a positive operator on $\mathscr{H}$, respectively.

Proof. For $T \in \mathscr{B}(\mathscr{H})$ we have, from the reproducing property of $K$, that for every $f \in \mathscr{H}$ and every $y \in X$,

$$
\{T f\}(y)=\langle T f, K(\cdot, y)\rangle=\left\langle f, T^{*} K(\cdot, y)\right\rangle .
$$

Thus $L_{T}(x, y)=\left\{T^{*} K(\cdot, y)\right\}(x)$ is the unique kernel which represents $T$. Another application of the reproducing property gives

$$
\overline{L_{T}(x, y)}=\left\langle\overline{T^{*} K(\cdot, y), K(\cdot, x)}\right\rangle=\langle T K(\cdot, x), K(\cdot, y)\rangle=\{T K(\cdot, x)\}(y)
$$

and thus $L_{T}(x, y)=\overline{\{T K(\cdot, x)\}(y)}$. In particular, $L_{T^{*}}(x, y)=\overline{L_{T}(y, x)}$.

To verify that $L_{T}$ is indeed a kernel subordinate to $K$, let $\left\{x_{i}\right\}$ be a finite sequence of points in $X$ and let $\left\{\alpha_{i}\right\},\left\{\beta_{i}\right\}$ be corresponding sequences of complex numbers. Set

$$
f=\sum \bar{\alpha}_{i} K\left(\cdot, x_{i}\right), \quad g=\sum \bar{\beta}_{i} K\left(\cdot, x_{i}\right)
$$

Then

$$
\langle T f, g\rangle=\sum \bar{\alpha}_{i} \beta_{j}\left\langle T K\left(\cdot, x_{i}\right), K\left(\cdot, x_{j}\right)\right\rangle=\sum \bar{\alpha}_{i} \beta_{j} \overline{L_{T}\left(x_{i}, x_{j}\right)}
$$


Thus the inequality (2.1) becomes $|\langle T f, g\rangle| \leqslant c\|f\|\|g\|$, which clearly holds in our case with $c=\|T\|$. Moreover, since functions of the form $\sum \alpha_{i} K\left(\cdot, x_{i}\right)$ are dense in $\mathscr{H}$, it follows that

$$
\left\|L_{T}\right\|=\sup \{|\langle T f, g\rangle| /\|f\| \cdot\|g\|: f, g \in \mathscr{H} \backslash\{0\}\}=\|T\| .
$$

Clearly, $L_{T}$ is hermitian on $X$ if and only if $T \in \mathscr{B}(\mathscr{H})$ is selfadjoint. Moreover, taking $f=g=\sum \bar{\alpha}_{i} K\left(\cdot, x_{i}\right)$ in the above gives

$$
\langle f, T f\rangle=\sum \alpha_{i} \bar{\alpha}_{j} L_{T}\left(x_{i}, x_{j}\right)
$$

so it follows that $L_{T}$ is positive-definite on $X$ if and only if $T \in \mathscr{B}(\mathscr{H})$ is a positive operator.

It remains to be shown that every kernel $L \in \mathscr{S}(K)$ represents some operator $T \in \mathscr{B}(\mathscr{H})$. Let $L$ be such a kernel. We construct the corresponding operator at first on a dense subspace $\mathscr{H}_{0}$ of $\mathscr{H}$ consisting of finite sums of the form $\sum \alpha_{i} K\left(\cdot, x_{i}\right)$. Define $S_{0}: \mathscr{H}_{0} \rightarrow \mathscr{H}$ by

$$
S_{0}\left(\sum \alpha_{i} K\left(\cdot, x_{i}\right)\right)=\sum \alpha_{i} L\left(\cdot, x_{i}\right) .
$$

Note that it follows from (2.1) that $S_{0}\left(\sum \alpha_{i} K\left(\cdot, x_{i}\right)\right)$ is a well-defined function on $X$, and by Lemma 2.3 it lies in $\mathscr{H}$. Letting $f=\sum \bar{\alpha}_{i} K\left(\cdot, x_{i}\right)$ and $g=\sum \bar{\beta}_{i} K\left(\cdot, x_{i}\right)$ as in the first part of the proof, we see from (2.1) that

$$
\left|\left\langle S_{0} f, g\right\rangle\right|^{2} \leqslant\|L\|\|f\|\|g\|
$$

for every $f, g \in \mathscr{H}_{0}$. Thus $S_{0}$ extends uniquely as a continuous linear operator $S$ on $\mathscr{H}$. Thus, setting $T=S^{*}$ we see that $T \in \mathscr{B}(\mathscr{H})$ and, for every $f \in \mathscr{H}$ and all $y \in X$,

$\{T f\}(y)=\langle T f, K(\cdot, y)\rangle=\left\langle f, T^{*} K(\cdot, y)\right\rangle=\left\langle f, S_{0} K(\cdot, y)\right\rangle=\langle f, L(\cdot, y)\rangle$. Hence $L=L_{T}$. Finally, for any $T_{1}, T_{2} \in \mathscr{B}(\mathscr{H})$ and any $\alpha_{1}, \alpha_{2} \in \mathrm{C}$ we have $L_{\left(\alpha_{1} T_{1}+\alpha_{2} T_{2}\right)}=\bar{\alpha}_{1} L_{T_{1}}+\bar{\alpha}_{2} L_{T_{2}}$, showing that the mapping is indeed conjugate-linear. The proof is now complete.

2.6. CoROLlaRY. A kernel $L$ on $X$ is in $\mathscr{S}(K)$ if and only if the following conditions hold:

(i) For every $x \in X$, the functions $L(\cdot, x)$ and $\overline{L(x, \cdot)}$ are in $\mathscr{H}$.

(ii) The operator $T$ defined by $\{T f\}(y)=\langle f, L(\cdot, y)\rangle$ is a member of $\mathscr{B}(\mathscr{H})$.

It follows from the preceding theorem that the space $\mathscr{S}(K)$ has a natural structure of a Banach algebra with involution, obtained by pulling back the operations in $\mathscr{B}(\mathscr{H})$. Thus we define the product of two kernels $L_{1}, L_{2} \in \mathscr{S}(K)$ by $\left(L_{1} L_{2}\right)(x, y)$ $=\left\langle L_{1}(\cdot, y), \overline{L_{2}(x, \cdot)}\right\rangle$. The adjoint $L^{*}$ of $L \in \mathscr{S}(K)$ is given, as before, by $L^{*}(x, y)=\overline{L(y, x)}$.

In the following theorem, it may be helfpul to keep in mind the case of the Bergman or Szegö kernel of a domain.

2.7. TheOREM. Let $E$ be an arbitrary subset of $X$ and let $K_{E}$ denote the restriction of $K$ to $E \times E$. Let $L$ be a kernel on $E$ which is subordinate to $K_{E}$, i.e. $L \in \mathscr{S}\left(K_{E}\right)$. Then there is a unique kernel $\tilde{L}$ on $X$ with the following properties:

(i) $\tilde{L} \in \mathscr{S}(K)$. 
(ii) $\left.L\right|_{E \times E}=L$.

(iii) For every $f \in \mathscr{H}$ with $\left.f\right|_{E}=0$, and every $y \in X$,

$$
\langle f, \tilde{L}(\cdot, y)\rangle=\langle f, \tilde{L}(y, \cdot)\rangle=0 \text {. }
$$

Moreover, if $L$ is hermitian or positive-definite on $E$, then the same is true of $E$ on $X$.

Proof. We denote by $\mathscr{H}_{E}$ the functional Hilbert space associated with $K_{E}$ and we note that $\mathscr{H}_{E}$ is precisely the set of restrictions to $E$ of functions in $\mathscr{H}$ (see Aronszajn [1]). If we let $R: \mathscr{H} \rightarrow \mathscr{H}_{E}$ be the restriction map then for any $f \in \mathscr{H}_{E}$ and any $y \in E$ we have

$$
\left\{R^{*} f\right\}(y)=\left\langle R^{*} f, K(\cdot, y)\right\rangle=\left\langle f, K_{E}(\cdot, y)\right\rangle_{E}=f(y) .
$$

Here $\langle,\rangle_{E}$ denotes the inner product in $\mathscr{H}_{E}$. Thus $R R^{*}$ is the identity operator on $\mathscr{H}_{E}$ and $R^{*} R$ is the orthogonal projection on $\mathscr{R}\left(R^{*}\right)=\mathscr{N}(R)^{\perp}$, the range of $R^{*}$ (identified as the orthogonal complement in $\mathscr{H}$ of the null-space of $R$ ).

Let $T \in \mathscr{B}\left(\mathscr{H}_{E}\right)$ be the linear operator on $\mathscr{H}_{E}$ represented by $L$ and set $\tilde{T}=R^{*} T R$. Clearly $\tilde{T}$ is selfadjoint or positive on $\mathscr{H}$ if and only if the same is true for $T$ on $\mathscr{H}_{E}$. Now let $\tilde{L}$ be the kernel on $X$ which represents $\tilde{T}$, i.e. $\tilde{L}(x, y)=\left\{\tilde{T}^{*} K(\cdot, y)\right\}(x)$. Then $\tilde{L}$ clearly satisfies property (iii) since, for any $y \in X, \tilde{L}(\cdot, y)$ and $\tilde{\tilde{L}(y, \cdot)}$ are both in the range of $R^{*}$. Moreover, if $x, y \in E$ then

$$
\begin{aligned}
\tilde{L}(x, y) & =\left\{R^{*} T^{*} R K(\cdot, y)\right\}(x)=\left\{R^{*} T^{*} K_{E}(\cdot, y)\right\}(x) \\
& =\left\{R^{*} L(\cdot, y)\right\}(x)=L(x, y) .
\end{aligned}
$$

Thus $\tilde{L}$ satisfies properties (i)-(iii). The assertions concerning hermitian and positive-definite kernels follow from the corresponding properties of the operator $\tilde{T}$.

It remains to show that the kernel $\tilde{L}$ is uniquely determined by properties (i)-(iii). Thus, let $\tilde{L}$ be any kernel satisfying (i)-(iii) and let $\tilde{T}$ be the associated operator. We will show that $\tilde{T}=R^{*} T R$. From (ii) we have for every $f \in \mathscr{H}_{E}$ and every $y \in X$,

$$
\begin{aligned}
\{T f\}(y) & =\langle f, L(\cdot, y)\rangle_{E}=\left\langle R R^{*} f, R \tilde{L}(\cdot, y)\right\rangle_{E} \\
& =\left\langle R^{*} f, R^{*} R \tilde{L}(\cdot, y)\right\rangle=\left\langle R^{*} f, \tilde{L}(\cdot, y)\right\rangle=\left\{\tilde{T} R^{*} f\right\}(y) .
\end{aligned}
$$

Hence we have $R \tilde{T} R^{*}=T$, and so $R^{*} T R=P \tilde{T} P$ where $P=R^{*} R$ is the orthogonal projection on $\mathscr{R}\left(R^{*}\right)=\mathscr{N}(R)^{\perp}$. Thus, to complete the proof, it suffices to show that $\mathscr{N}(\tilde{T}) \supset \mathscr{N}(R)$ and $\mathscr{R}(\tilde{T}) \subset \mathscr{N}(R)^{\perp}$. But these inclusions are immediate from (iii) since $\tilde{L}(\cdot, y)=\tilde{T}^{*} K(\cdot, y), \overline{\tilde{L}(y, \cdot)}=\tilde{T} K(\cdot, y)$, and the functions $K(\cdot, y)$ are linearly dense in $\mathscr{H}$. This completes the proof.

A subset $E$ of $X$ is a set of uniqueness for a family $\mathscr{F}$ of functions on $X$ if the restriction mapping $\left.f \mapsto f\right|_{E}$ is injective on $\mathscr{F}$.

2.8. Corollary. Let $E$ be a set of uniqueness for $\mathscr{H}$ and let $L$ be a kernel on $X$ which is subordinate to $K$, i.e. $L \in \mathscr{S}(K)$. If the restriction of $L$ to $E \times E$ is positive-definite, then $L$ is positive-definite on $X$.

The following interpolation result for functions follows immediately from Theorem 2.2. 
2.9. THEOREM. Let $E$ be an arbitrary subset of $X$ and let $f$ be a function on $E$ such that the kernel $c^{2} K(x, y)-f(x) \overline{f(y)}$ is positive-definite on $E$ for some nonnegative constant $c$. Then there is a function $f \in \mathscr{H}$ such that $\left.F\right|_{E}=f$ and such that the kernel $c^{2} K(x, y)-F(x) \overline{F(y)}$ is positive-definite on $X$.

Proof. By Theorem 2.2, $f$ is in the functional Hilbert space $\mathscr{H}_{E}$ associated with $K_{E}=\left.K\right|_{E \times E}$. Using the notation of the proof of Theorem 2.7, we set $F=R^{*} f$. Then $\left.F\right|_{E}=R R^{*} f=f$, and

$$
\|F\|^{2}=\left\langle R^{*} f, R^{*} f\right\rangle=\left\langle R R^{*} f, f\right\rangle_{E}=\|f\|_{E}^{2} \leqslant c^{2} .
$$

By a second application of Theorem 2.2, we conclude that $c^{2} K(x, y)=F(x) \overline{F(y)}$ is positive-definite on $X$.

We now prove a version of the preceding theorem which is valid for kernels which are not positive-definite.

2.10. Corollary. Let $L$ be a kernel on $X$ which is subordinate to $K$, i.e. $L \in \mathscr{S}(K)$, and let $E$ be a subset of $X$. Let $f$ be a function on $E$ such that the kernel $c^{2} L(x, y)-f(x) \overline{f(y)}$ is positive-definite on $E$ for some nonnegative constant $c$. Then there is a function $F$ in $\mathscr{H}$ with $\left.F\right|_{E}=f$ and $\|F\| \leqslant c \sqrt{\|L\|}$.

Proof. Our hypothesis clearly implies that $L$ is positive-definite on $E$. By Theorem 2.7 there is a positive-definite kernel $\tilde{L} \in \mathscr{S}(K)$ on $X$ which agrees with $L$ on $E \times E$ and with $\|\tilde{L}\| \leqslant\|L\|$. Let $\tilde{\mathscr{H}}$ be the functional Hilbert space associated with $\tilde{L}$. By Theorem 2.9 there is a function $F$ in $\tilde{\mathscr{H}}$ such that $c^{2} \tilde{L}(x, y)-F(x) \overline{F(y)}$ is positive-definite on $X$. Then by definition of $\|\tilde{L}\|$ we have that $\|\tilde{L}\| K(x, y)-\tilde{L}(x, y)$ is positive-definite. Thus it follows that $c^{2}\|\tilde{L}\| K(x, y)-F(x) \overline{F(y)}$ is positive-definite. By Theorem 2.2, we deduce that $F \in \mathscr{H}$ and $\|F\| \leqslant c \sqrt{\|\tilde{L}\|} \leqslant c \sqrt{\|L\|}$. This concludes the proof.

We close this section by introducing the following concept. A function $F$ on $X$ is said to have the multiplication-property with respect to $K$ if the kernel $K_{F}(x, y)$ $=\overline{F(y)} K(x, y)$ on $X$ is subordinate to $K$, i.e. $K_{F} \in \mathscr{S}(K)$. In this case the kernel $K_{F}$ represents an operator $M_{F} \in \mathscr{B}(\mathscr{H})$, known as the mulitplication-operator due to $F$. It follows from Theorem 2.5 that $M_{F} g=F g$ for all $g \in \mathscr{H}$ (see also Burbea and Masani $[9,10]$ for a vectorial version of these concepts).

2.11. THEOREM. Let $F$ be a function on $X$, having the multiplication-property with respect to $K$, and let $c$ be a nonnegative constant. Then $K(x, y)\left(c^{2}-F(x) \overline{F(y)}\right)$ is positive-definite on $X$ if and only if $\left\|M_{F}\right\| \leqslant c$.

Proof. According to Theorem 2.5 the operator on $\mathscr{H}$ associated with the above kernel is $c^{2} I-M_{F} M_{F}^{*}$, where $I$ is the identity operator on $\mathscr{H}$. Thus it follows that the kernel $K(x, y)\left(c^{2}-F(x) \overline{F(y)}\right)$ is positive-definite on $X$ if and only if $c^{2} I-$ $M_{F} M_{F}^{*}$ is a positive operator on $\mathscr{H}$. However, the latter is equivalent to $\left\|M_{F}\right\| \leqslant c$, and the theorem follows.

We remark that the last theorem shows in particular that

$$
\sup \{|F(x)|: x \in X, K(x, x) \neq 0\} \leqslant\left\|M_{F}\right\|
$$

whenever $F$ has the multiplication-property with respect to $K$. 
Finally, we should remark that the results of this and the next section could also be formulated with positive-definiteness replaced by conditional positive-definiteness. This applies, in particular, to Theorems 3.4 and 3.5. A kernel $K$ on $X$ is said to be conditionally positive-definite on $X$ if for any finite sequences $\left\{x_{i}\right\}$ in $X$ and $\left\{\alpha_{i}\right\}$ in $\mathrm{C}$ with $\sum \alpha_{i}=0$, we have $\sum \alpha_{i} \bar{\alpha}_{j} K\left(x_{i}, x_{j}\right) \geqslant 0$. This assertion follows from the simple observation that, for any point $x_{0} \in X$, the kernel $K$ is conditionally positive-definite on $X$ if and only if the kernel $K(x, y)-K\left(x_{0}, y\right)-K\left(x, x_{0}\right)+$ $K\left(x_{0}, x_{0}\right)$ is positive-definite on $X$. For these matters, we refer to Donoghue [11, p. 135], and to FitzGerald and Horn [16].

3. Interpolation theorems for holomorphic functions. In this section we specialize to spaces of holomorphic functions. If $D$ is a domain in $\mathbf{C}^{n}$ (or a complex manifold), a kernel $K$ on $D$ will be called sesqui-holomorphic if for each $z \in D$ the functions $K(\cdot, z)$ and $\overline{K(z, \cdot)}$ are holomorphic on $D$. Thus, a reproducing kernel associated with a functional Hilbert space of holomorphic functions is sesqui-holomorphic, and conversely, any sesqui-holomorphic positive-definite kernel on $D$ is the reproducing kernel of a functional Hilbert space of holomorphic functions on $D$. By $\mathcal{O}(D)$ we denote the algebra of holomorphic functions on $D$. We will be especially interested in functional Hilbert spaces of the form

$$
\mathscr{H}_{\mu}(D)=\left\{f \in \mathcal{O}(D):\|f\|_{\mu}^{2} \equiv \int_{D}|f|^{2} d \mu<\infty\right\}
$$

where $\mu$ is some positive measure on $D$. We will not address the question of which positive measures give rise to functional Hilbert spaces on $D$. Suffice it to say that $\mathscr{H}_{\mu}(D)$ is a functional Hilbert space on $D$ if for any compact subset $B$ of $D$ there exists a positive constant $c=c(B)$ such that $d \mu(z) \geqslant c(B) d v(z)$ for all $z \in B$, where $d v$ is the euclidean volume element. This follows easily from the mean value property of holomorphic functions. In particular, $\mathscr{H}_{\mu}(D)$ is a functional Hilbert space on $D$ if $d \mu=\phi d v$, where $\phi$ is a positive continuous function on $D$. In this case we will call $\mathscr{H}_{\mu}(D)$ a weighted Bergman space and we denote it by $A_{\phi}^{2}(D)$.

Let us observe that if $K_{\mu}$ is the reproducing kernel associated with the functional Hilbert space $\mathscr{H}_{\mu}(D)$, then it follows from Corollary 2.6 that a sufficient condition for a sesqui-holomorphic kernel $L$ on $D$ to be subordinate to $K_{\mu}$ is that $L$ is a "Hilbert-Schmidt kernel" namely,

$$
\int_{D} \int_{D}|L(z, \zeta)|^{2} d \mu(z) d \mu(\zeta)<\infty
$$

Unless otherwise specified, we shall assume that $\mu$ is a positive measure on $D$ such that $\mathscr{H}_{\mu}=\mathscr{H}_{\mu}(D)$ is a functional Hilbert space on $D$. In this case $K_{\mu}$ denotes the reproducing kernel of $\mathscr{H}_{\mu}$.

The next result is a special case of Theorem 2.7.

3.1. TheOREM. Let $D$ be a domain in $\mathbf{C}^{n}$ and let $L$ be a kernel on a subset $E$ of $D$ which is subordinate to $K_{\mu}$ on $E$. Then there is a sesqui-holomorphic kernel $\tilde{L}$ on $D$, subordinate to $K_{\mu}$, i.e. $\tilde{L} \in \mathscr{S}\left(K_{\mu}\right)$, such that $\left.\tilde{L}\right|_{E \times E}=L$ and $\|\tilde{L}\|=\|L\|$. Moreover, if 
$L$ is hermitian or positive-definite on $E$, then $L$ can be chosen to be hermitian or positive-definite, respectively, on $D$.

3.2. Corollary. Let $D$ be a domain in $\mathbf{C}^{n}$ and let $E$ be a subset of $D$ which is also a set of uniqueness for $\mathscr{H}_{\mu}$. Let $L$ be a kernel on $D$ which is subordinate to $K_{\mu}$, i.e. $L \in \mathscr{S}\left(K_{\mu}\right)$. If $L$ is hermitian or positive-definite on $E$, then the same is true on $D$.

Proof. By Theorem 3.1, $\left.L\right|_{E \times E}$ has an extension $\tilde{L}$ to $D \times D$ with the desired properties. But since $E$ is a set of uniqueness for $\mathscr{H}_{\mu}$, we must have $\tilde{L}=L$.

We now formulate a version of the last corollary which does not involve the auxiliary Hilbert space $H_{\mu}$. Various versions of this result have been obtained by FitzGerald [14], Donoghue [12], and FitzGerald and Horn [16].

3.3. THEOREM. Let $L$ be a sesqui-holomorphic kernel on a domain $D$ in $\mathbf{C}^{n}$ and let $E$ be a set of uniqueness for $\mathcal{O}(D)$. If $\left.L\right|_{E \times E}$ is hermitian or positive-definite, then the same is true of $L$.

Proof. Choose a positive continuous function $\phi$ on $D$ such that

$$
\int_{D} \int_{D}|L(z, \zeta)|^{2} \phi(z) \phi(\zeta) d v(z) d v(\zeta)<\infty
$$

Then $L$ is subordinate to the reproducing kernel $K_{\phi}$ of the weighted Bergman space $A_{\phi}^{2}(D)$. Thus the result follows from Corollary 3.2 .

We now turn our attention to interpolation theorems for holomorphic functions. The next theorem is essentially due to FitzGerald [14] in the special case that $E$ is a set of uniqueness for $\mathcal{O}(D)$.

3.4. THEOREM. Let $K$ be a sesqui-holomorphic kernel on a domain $D$ in $\mathbf{C}^{n}$. Let $f$ be a function on a subset $E$ of $D$ such that for some nonnegative constant $c$ the kernel $c^{2} K(z, \zeta)-f(z) \overline{f(\zeta)}$ is positive-definite on $E$. Then there is a holomorphic function $F$ on $D$ with $\left.F\right|_{E}=f$. Moreover, if $K$ is positive-definite on $D$ or if $E$ is a set of uniqueness for $\mathcal{O}(D)$ then $F$ can be chosen so that the kernel $c^{2} K(z, \zeta)-F(z) \overline{F(\zeta)}$ is positive-definite on $D$.

Proof. If $K$ is positive-definite, then the result follows from Theorem 2.10. Moreover, if $E$ is a set of uniqueness for $\mathcal{O}(D)$ then it follows from Theorem 3.3 that $K$ is positive-definite on $D$. To complete the proof we must show that $f$ has a holomorphic extension to $D$ if $K$ is not assumed to be positive-definite. Choose a positive continuous function $\phi$ on $D$ so that

$$
\int_{D} \int_{D}|L(z, \zeta)|^{2} \phi(z) \phi(\zeta) d v(z) d v(\zeta)<\infty
$$

Then $K$ is subordinate to the reproducing kernel $K_{\phi}$ of $A_{\phi}^{2}(D)$. It follows from Corollary 2.10 that there is a function $F \in A_{\phi}^{2}(D)$ with $\left.F\right|_{E} f$. The proof is now complete. 
To illustrate the utility of Theorem 3.4 we introduce a family of kernels on the unit polydisk $\Delta^{n}$ in $\mathbf{C}^{n}$. For any $n$-tuple $\underline{\alpha}=\left(\alpha_{1}, \ldots, \alpha_{n}\right)$ of nonnegative numbers we set

$$
K_{\underline{\alpha}}(z, \zeta)=\prod_{j=1}^{n}\left(1-z_{j} \bar{\zeta}_{j}\right)^{-\alpha_{j}}
$$

Note that $\log K_{\alpha}$ is a sum of positive-definite kernels on $\Delta^{n}$, so it follows that $K_{\alpha}$ is positive-definite on $\Delta^{n}$. We denote by $H_{\alpha}\left(\Delta^{n}\right)$ the functional Hilbert space of holomorphic functions on $\Delta^{n}$ with $K_{\alpha}$ as its reproducing kernel. If $\underline{\alpha}=\underline{1}$ (i.e. when $\alpha_{j}=1$ for $\left.j=1, \ldots, n\right)$ then $H_{\alpha}\left(\Delta^{n}\right)$ is the usual Hardy-Szegö space, i.e.

$$
H_{\underline{1}}\left(\Delta^{n}\right)=H^{2}\left(\Delta^{n}\right)=\left\{f \in \mathcal{O}\left(\Delta^{n}\right):\|f\|_{\underline{1}}^{2}=(2 \pi)^{-n} \sup _{0 \leqslant r<1} \int_{(\partial \Delta)^{n}}|f(r z)|^{2}|d z|<\infty\right\},
$$

where $d z=d z_{1} \cdots d z_{n}$. If, on the other hand, $\underline{\alpha}>\underline{1}$ (i.e. $\alpha_{j}>1$ for $j=1, \ldots, n$ ) then $H_{\alpha}\left(\Delta^{n}\right)$ is a weighted Bergman space

$$
\begin{aligned}
& H_{\underline{\alpha}}\left(\Delta^{n}\right)=\left\{f \in \mathcal{O}\left(\Delta^{n}\right):\right. \\
& \left.\quad\|f\|_{\underline{\alpha}}^{2}=\pi^{-n} \prod_{j=1}^{n}\left(\alpha_{j}-1\right) \int_{\Delta^{n}}|f(z)|^{2} \prod_{j=1}^{n}\left(1-\left|z_{j}\right|^{2}\right)^{\alpha_{j}-2} d v(z)<\infty\right\} .
\end{aligned}
$$

Let $R: \mathcal{O}\left(\Delta^{n}\right) \rightarrow \mathcal{O}(\Delta)$ be the diagonal restriction mapping defined by $\{R f\}(\omega)=$ $f(\omega, \ldots, \omega)$. It follows from Theorem 3.4 that $R$ is a norm-decreasing map of $H_{\alpha}\left(\Delta^{n}\right)$ onto $H_{|\underline{\alpha}|}(\Delta)$, where $|\underline{\alpha}|=\sum_{j=1}^{n} \alpha_{j}$. Moreover, there is a linear isometry $E_{\alpha}: H_{\lfloor\alpha \mid}(\bar{\Delta}) \rightarrow$ $H_{\underline{\alpha}}\left(\Delta^{n}\right)$ such that $R E_{\underline{\alpha}}=1$. In particular, $R$ maps the Hardy-Szegö space $H^{2}\left(\Delta^{n}\right)$ onto the weighted Bergman space $H_{n}(\Delta) \equiv A_{\phi}^{2}(\Delta)$ with weight

$$
\phi(\omega)=\pi^{-1}(n-1)\left(1-|\omega|^{2}\right)^{n-2} .
$$

For other approaches to the problem, see Rudin [26, p. 53], Duren and Shields [13], Horowitz and Oberlin [18], Moulin and Rosay [22], and Shapiro [28].

Considerations similar to the above apply also to the unit ball $B_{n}$ in $\mathbf{C}^{n}$. For $s>0$ we define the kernel $K_{s}$ on $B_{n}$ by

$$
K_{s}(z, \zeta)=(1-\langle z, \zeta\rangle)^{-s}
$$

Then $K_{s}$ is positive-definite on $B_{n}$ (see also Burbea [7]). We denote by $H_{s}\left(B_{n}\right)$ the associated functional Hilbert space of Holomorphic functions on $B_{n}$. The norm for this space is defined via fractional-derivations. For $f \in \mathcal{O}\left(B_{n}\right)$, we have, using multinomial notation,

$$
f(z)=\sum_{\alpha} a_{\alpha} z^{\alpha} ; \quad a_{\alpha} \in \mathbf{C}, z \in B_{n},
$$

where $\alpha=\left(\alpha_{1}, \ldots, \alpha_{n}\right)$ is an $n$-tuple of nonnegative integers and $z^{\alpha}$ is the abbreviation for $z_{1}^{\alpha_{1}} \cdots z_{n}^{\alpha_{n}}$. For any $r, 0 \leqslant r \leqslant 1$, the fractional-derivation of order $r$ of $f$, $f^{\langle r\rangle}$, is defined by

$$
f^{\langle r\rangle}(z)=\sum_{\alpha}\left\{\alpha_{1} \cdots \alpha_{n}\right\}^{r} a_{\alpha} z^{\alpha-\underline{1}+[1-r] \underline{1}}
$$

where $[1-r]$ is the integer value of $1-r$. Thus it follows that $f^{\langle 0\rangle}=f, f^{\langle 1\rangle}=\partial_{z_{1}}$ $\cdots \partial_{z_{n}} f$ and

$$
f^{\langle 1 / 2\rangle}(z)=\sum_{\alpha} \sqrt{\alpha_{1} \cdots \alpha_{n}} a_{\alpha} z^{\alpha-1} .
$$


We also define

$$
\|f\|_{s}^{2}=\pi^{-n} \int_{B_{n}}\left|f^{\langle 1 / 2\rangle}(z)\right|^{2}\left(1-|z|^{2}\right)^{s-1} d v(z) .
$$

With this norm, we have

$$
H_{s}\left(B_{n}\right)=\left\{f \in \mathcal{O}\left(B_{n}\right):\|f\|_{s}<\infty\right\} .
$$

For $s=n$ the norm in $H_{s}\left(B_{n}\right)$ can also be realized as

$$
\|f\|_{n}^{2}=\frac{(n-1) !}{2 \pi^{n}} \sup _{0 \leqslant r<1} \int_{\partial B_{n}}|f(r z)|^{2} d \sigma(z),
$$

and thus $H_{n}\left(B_{n}\right)$ is the usual Hardy-Szegö space $H^{2}\left(B_{n}\right)$. Similarly, for $s>n$ the norm in $H_{s}\left(B_{n}\right)$ can be realized as

$$
\|f\|_{s}^{2}=\frac{1}{\pi^{n}} \frac{\Gamma(s)}{\Gamma(s-n)} \int_{B_{n}}|f(z)|^{2}\left(1-|z|^{2}\right)^{s-n-1} d v(z)
$$

and thus $H_{s}\left(B_{n}\right), s>n$, is a weighted Bergman space $A_{\phi}^{2}\left(B_{n}\right)$ with weight $\phi(z)=$ $\pi^{-n}(\Gamma(s) / \Gamma(s-n))\left(1-|z|^{2}\right)^{s-n-1}$.

Let $k$ be an integer, $1 \leqslant k \leqslant n$, and consider the unit ball $B_{k}$ of $C^{k}$. We define a restriction mapping $R_{k}: \mathcal{O}\left(B_{n}\right) \rightarrow \mathcal{O}\left(B_{k}\right)$ by $R_{k} f(z)=f(z, 0)$ for $z \in B_{k}$ and $0 \in$ $\mathrm{C}^{n-k}$. From Theorem 3.4 it follows that $R_{k}$ is a norm-decreasing map of $H_{s}\left(B_{n}\right)$ onto $H_{s}\left(B_{k}\right)$. In particular, for $1 \leqslant k \leqslant n, R_{k}$ maps the Hardy-Szegö space $H^{2}\left(B_{n}\right)$ onto the weighted Bergman space $A_{\phi}^{2}\left(B_{k}\right)$ with weight $\phi$, given by

$$
\phi(z)=\pi^{-k}(n-k)(n-k+1) \cdots(n-1)\left(1-|z|^{2}\right)^{n-k-1} .
$$

Moreover, the extension mapping $E_{k}: \mathcal{O}\left(B_{k}\right) \rightarrow \mathcal{O}\left(B_{n}\right)$ definea by $\left\{E_{k} f\right\}\left(z_{1}, \ldots, z_{n}\right)$ $=f\left(z_{1}, \ldots, z_{k}\right)$ is an isometric embedding of $H_{s}\left(B_{k}\right)$ into $H_{s}\left(B_{n}\right)$ (compare Rudin [27, p. 127]).

Yet another application is obtained by applying Theorem 3.4 to the Hilbert space $\mathscr{F}_{q}\left(\mathbf{C}^{n}\right), q>0$, of all entire functions $f$ on $\mathbf{C}^{n}$ satisfying

$$
\|f\|_{q}^{2}=\frac{q^{n}}{\pi^{n}} \int_{\mathbf{C}^{n}}|f(z)|^{2} e^{-q|z|^{2}} d v(z)<\infty .
$$

This is a functional Hilbert space on $\mathbf{C}^{n}$ with the reproducing kernel $K_{q}(z, \zeta)=$ $e^{q\langle z, \zeta\rangle}$ and is known as the Fischer space of order $q$ (see also Burbea [7]). Let $V$ be any linear subspace of $\mathbf{C}^{n}$ of complex dimension $k, 1 \leqslant k \leqslant n$, which we identify, via a unitary isomorphism with $\mathbf{C}^{k}$. Let $R_{k}: \mathcal{O}\left(\mathbf{C}^{n}\right) \rightarrow \mathcal{O}(V)$ be the restriction mapping $R_{k} f=\left.f\right|_{V}$, then Theorem 3.4 implies that $R_{k}$ is a norm-decreasing map of $\mathscr{F}_{q}\left(\mathbf{C}^{n}\right)$ onto $\mathscr{F}_{q}\left(\mathbf{C}^{k}\right)$. Again, the extension map $E_{k}: \mathcal{O}\left(\mathbf{C}^{k}\right) \rightarrow \mathcal{O}\left(\mathbf{C}^{n}\right)$ defined by

$$
\left\{E_{k} f\right\}\left(z_{1}, \ldots, z_{n}\right)=f\left(z_{1}, \ldots, z_{k}\right)
$$

is an isometric embedding of $\mathscr{F}_{q}\left(\mathbf{C}^{k}\right)$ into $\mathscr{F}_{q}\left(\mathbf{C}^{n}\right)$.

Our next result is in the spirit of the classical Pick-Nevanlinna interpolation theorem. A similar but more restrictive problem has also been considered by Hahn [17]. 
3.5. Theorem. Let $D$ be a domain in $\mathbf{C}^{n}$ and let $K$ be a positive-definite sesqui-holomorphic kernel on $D$ with the property that $K(z, z) \neq 0$ for every $z \in D$. Let $E$ be a set of uniqueness for $\mathcal{O}(D)$ and let $f$ be a function on $E$ such that $K(z, \zeta)\left(c^{2}-f(z) \overline{f(\zeta)}\right)$ is positive-definite on $E$ for some nonnegative constant $c$. Then there is a unique holomorphic function $F$ on $D$ with $\left.F\right|_{E}=f$. Moreover, $K(z, \zeta)\left(c^{2}-F(z) \overline{F(\zeta)}\right)$ is positive-definite on $D$. In particular, $|F(z)| \leqslant c$ for every $z \in D$.

Proof. Let $\mathscr{H}$ be the functional Hilbert space associated with $K$, and let $g \in \mathscr{H}$, $g$ not identically zero. By Theorem 2.2 the kernel $\|g\|^{2} K(z, \zeta) f(z) \overline{f(\zeta)}-$ $f(z) g(z) \overline{f(\zeta) g(\zeta)}$ is positive-definite on $E$, and hence by our hypothesis, $c^{2}\|g\|^{2} K(z, \zeta)-f(z) g(z) \overline{f(\zeta) g(\zeta)}$ is positive-definite on $E$. By Theorem 3.4 there is a function $G \in \mathscr{H}$ with $\left.G\right|_{E}=f g$. Let $V_{g}=\{z \in D: g(z)=0\}$ and set $F_{g}(z)=$ $G(z) / g(z)$ for $z \in D \backslash V_{g}$. Then clearly $F_{g}$ is holomorphic on $D \backslash V_{g}$ and $F_{g}$ agrees with $f$ on $E \backslash V_{g}$. Now, let $h$ be another nonzero member of $\mathscr{H}$ and construct the functions $H$ and $F_{h}$ as above. We will show that $F_{g}=F_{h}$ on $D \backslash\left(V_{g} \cap V_{h}\right)$. Since $F_{g}=F_{h}=f$ on $E \backslash\left(V_{g} \cap V_{h}\right)$, it follows that $G h-H g=0$ on $E \backslash\left(V_{g} \cap V_{h}\right)$, and so $g h(G h-H g)=0$ on $E$. Since $E$ is a set of uniqueness, it follows that $G h-H g=0$ on $D$, and so $F_{g}=F_{h}$ on $D \backslash\left(V_{g} \cap V_{h}\right)$. Hence it follows that there is a function $F$ which is holomorphic on $D \backslash \bigcap\left\{V_{g}: g \in \mathscr{H}, g \neq 0\right\}$ and such that $\left.F\right|_{E}=f$. But since $K(z, z) \neq 0$ for all $z \in D$, we have $\bigcap\left\{V_{g}: g \in \mathscr{H}, g \neq 0\right\}=\varnothing$, so $F \in \mathcal{O}(D)$. By hypothesis, the sesqui-holomorphic kernel $K(z, \zeta)\left(c^{2}-F(z) \overline{F(\zeta)}\right)$ is positive-definite on $E$, hence as $E$ is a set of uniqueness for $\mathcal{O}(D)$, it is positive-definite on $D$ by Theorem 3.3. This concludes the proof.

Let $K$ be a positive-definite sesqui-holomorphic kernel on a domain $D$ in $\mathbf{C}^{n}$ and let $\mathscr{H}$ be the functional Hilbert space associated with $K$. Let $H^{\infty}(D)$ denote the Banach algebra of bounded holomorphic functions on $D$. The kernel $K$ is said to have the contraction-property if every $F \in H^{\infty}(D)$ has the multiplication-property with respect to $K$ with $\left\|M_{F}\right\| \leqslant\|F\|_{\infty}$. The kernel $K_{\mu}$ of $\mathscr{H}_{\mu}(D)$ as well as the Szegö kernel $S$ of $H^{2}(D)$ clearly possess the contraction-property. In this case, for $F \in H^{\infty}(D), M_{\bar{F}}$ (pointwise multiplication by $\bar{F}$ ) is a continuous linear operator on $L^{2}(d \mu)$ and $M_{F}^{*}=P_{\mu} M_{\bar{F}}$ where $P_{\mu}$ denotes the orthogonal projection of $L_{2}(d \mu)$ onto $\mathscr{H}_{\mu}(D)$; in the Szegö kernel case, $L^{2}(d \mu)$ is replaced by $L^{2}(\partial D)$ and $P_{\mu}$ by $\mathbf{S}$, the orthogonal projection of $L^{2}(\partial D)$ onto $H^{2}(\partial D)$ in the sense described in $\S 4$.

We now prove (cf. Burbea $[4,6]$ )

3.6. Proposition. Let $K$ be a positive-definite sesqui-holomorphic kernel on a domain $D$ on $\mathbf{C}^{n}$ which is not zero identically and let $F_{0}$ be a function on $D$, having the multiplication-property with respect to $K$. Assume that $F_{0}$ is continuous at each point of the variety $\{z \in D: K(z, z)=0\}$. Then $F_{0} \in H^{\infty}(D)$ and $\left\|F_{0}\right\|_{\infty} \leqslant\left\|M_{F}\right\|$. If , in additon, $K$ has the contraction-property then $\left\|M_{F_{0}}\right\|=\left\|F_{0}\right\|_{\infty}$. Moreover, the mapping $F \mapsto M_{F}$ is a linear isometry of $H^{\infty}(D)$ onto $\mathscr{M}(\mathscr{H})$, the Banach subalgebra of $\mathscr{B}(\mathscr{H})$ consisting of all multiplication-operators in $\mathscr{B}(\mathscr{H})$.

Proof. By assumption $F_{0} K(\cdot, z)$ is in $\mathscr{H}$ for every $z \in D$; in particular it is holomorphic in $D$ for every (fixed) $z \in D$. It follows, since $F_{0}$ is defined on $D$ and 
$K \not \equiv 0$, then $F_{0}$ is meromorphic on $D$. By Theorem 2.11, $\sup \left\{\left|F_{0}(z)\right|: z \in D_{0}\right\} \leqslant$ $\left\|M_{F_{0}}\right\|$ where $D_{0}=\{z \in D: K(z, z) \neq 0\}$. It follows by Riemann's theorem on removable singularities that $F_{0} \in \mathcal{O}(D)$ and $\left\|F_{0}\right\|<\left\|M_{F}\right\|$. If, in addition, $K$ possesses the contraction-property then the mapping $F \mapsto M_{F}$ is a well-defined linear operator from $H^{\infty}(D)$ into $\mathscr{M}(\mathscr{H})$ with $\left\|M_{F}\right\| \leqslant\|F\|_{\infty}$. It follows, by the first part of the theorem, that this mapping is onto $\mathscr{M}(\mathscr{H})$ and $\left\|M_{F}\right\|=\|F\|_{\infty}$. This concludes the proof.

3.7. COROllary. Let $K$ be a positive-definite sesqui-holomorphic kernel on a domain $D$ in $\mathbf{C}^{n}$, having the contraction-property and such that $K(z, z) \neq 0$ for all $z \in D$. Let $E$ be a set of uniqueness for $\mathcal{O}(D)$ and let $f$ be an arbitrary function on $E$. Then for any nonnegative constant $c$ the following conditions are equivalent:

(i) There is a unique $F \in H^{\infty}(D)$ with $\|F\|_{\infty} \leqslant c$ and $\left.F\right|_{E}=f$.

(ii) The kernel $K(z, \zeta)\left(c^{2}-f(z) \overline{f(\zeta)}\right)$ is positive-definite on $E$.

Proof. The implication (ii) $\Rightarrow$ (i) is a special case of Theorem 3.5. For the converse, we observe, since $K$ has the contraction-property, that for any $F \in H^{\infty}(D)$, the multiplication-operator $M_{F} \in \mathscr{M}(\mathscr{H})$ satisfies $\left\|M_{F}\right\| \leqslant\|F\|_{\infty}$ (in fact, $\left\|M_{F}\right\|=$ $\|F\|_{\infty}$ by Proposition 3.6). Condition (i), therefore, implies that $F$ has the multiplication-property with respect to $K$ and $\left\|M_{F}\right\| \leqslant c$. It follows from Theorem 2.11 that $K(z, \zeta)\left(c^{2}-F(z) \overline{F(\zeta)}\right)$ is positive-definite on $D$, and the proof is complete.

We remark that the classical interpolation theorem of Pick [24] characterizes the restrictions of holomorphic functions with values in a half-plane rather than a disk. One can verify easily, using a conformal mapping, that the two problems are equivalent. In fact, let $\Omega$ be any hyperbolic simply connected domain in the extended plane $\hat{\mathbf{C}}$ and let $\phi$ be a conformal mapping of $\Omega$ onto the unit disk $\Delta$. Then the Szegö kernel $S_{\Omega}$ of $\Omega$ satisfies

$$
S_{\Omega}(z, \zeta)=\left\{\phi^{\prime}(z) \overline{\phi^{\prime}(\zeta)}\right\}^{1 / 2} S_{\Delta}(\phi(z), \phi(\zeta)) ; \quad z, \zeta \in \Omega
$$

where

$$
S_{\Delta}(\omega, \tau)=\{2 \pi(1-\omega \bar{\tau})\}^{-1} ; \quad \omega, \tau \in \Delta,
$$

is the Szegö kernel of the unit disk.

3.8. COROllaRY. Let $K$ be a positive-definite sesqui-holomorphic kernel on a domain $D$ in $\mathbf{C}^{n}$ with the property that $K(z, z) \neq 0$ for every $z \in D$. Let $\Omega$ be a hyperbolic simply connected domain in $\mathbf{C}$ and let $S_{\Omega}$ be its Szegö kernel. Let $E$ be a set of uniqueness for $\mathcal{O}(D)$ and let $f$ be a function from $E$ into $\Omega$ such that the kernel $K(z, \zeta)\left[S_{\Omega}(f(z), f(\zeta))\right]^{-1}$ is positive-definite on $E$. Then there is a unique holomorphic function $F$ on $D$ with $\left.F\right|_{E}=f$. Moreover, we have $F(z) \in \Omega$ for $z \in D$, and the kernel $K(z, \zeta)\left[S_{\Omega}(F(z), F(\zeta))\right]^{-1}$ is positive-definite on $D$.

Proof. Let $\phi$ be a conformal mapping of $\Omega$ onto the unit disk and let $f_{1}=\phi \circ f$. We have, in accordance with (3.1)-(3.2), 


$$
\begin{aligned}
& K(z, \zeta)\left(1-f_{1}(z) \overline{f_{1}(\zeta)}\right) \\
& \quad=\frac{1}{2 \pi}\left\{\phi^{\prime}(f(z)) \overline{\phi^{\prime}(f(\zeta))}\right\}^{1 / 2} K(z, \zeta)\left[S_{\Omega}(f(z), f(\zeta))\right]^{-1}
\end{aligned}
$$

and thus $K(z, \zeta)\left(1-f_{1}(z) \overline{f_{1}(\zeta)}\right)$ is positive-definite on $E$. It follows from Theorem 3.5 that there exists a unique holomorphic function $F_{1}$ on $D$ with $\left.F_{1}\right|_{E}=f_{1}$ and $\left|F_{1}(z)\right| \leqslant 1$ for all $z \in D$. Moreover, since $\left|f_{1}(z)\right|<1$ for $z \in E$, it follows from the maximum modulus principle that $\left|F_{1}(z)\right|<1$ for all $z \in D$. Set $F=\phi^{-1} \circ F_{1}$. Then $F$ is holomorphic on $D$ with $\left.F\right|_{E}=f$ and $F(z) \in \Omega$ for all $z \in D$. Also, the sesqui-holomorphic kernel $K(z, \zeta)\left[S_{\Omega}(F(z), F(\zeta))\right]^{-1}$ is positive-definite on $E$ by hypothesis, so by Theorem 3.3 it is positive-definite on $D$. This concludes the proof.

The last corollary admits a stronger version if the boundary $\partial \Omega$ of the simply connected domain $\Omega$ is sufficiently smooth. According to a theorem of Warschawski [30], if $\partial \Omega$ is of class $C^{1}$ with a Dini-continuous normal, in particular if $\partial \Omega \in C^{1, \varepsilon}$ $(0<\varepsilon<1)$, then the conformal mapping $\phi: \Omega \rightarrow \Delta$ extends to a $C^{1}$-diffeomorphism of $\bar{\Omega}$ onto $\bar{\Delta}$ and there exist positive constants $a$ and $b$ such that

$$
0<a \leqslant\left|\phi^{\prime}(z)\right| \leqslant b<\infty, \quad z \in \bar{\Omega} .
$$

It follows that $\left\{S_{\Omega}(z, \zeta)\right\}^{-1}$ is well defined for all $z, \zeta \in \bar{\Omega}$ and is zero if and only if $z=\zeta \in \partial \Omega$.

3.9. Corollary. Let $K, D$ and $E$ be as in Corollary 3.8. Let $\Omega$ be a simply connected domain in $\hat{\mathbf{C}}$ such that $\partial \Omega$ is of class $C^{1}$ with a Dini-continuous normal and let $S_{\Omega}$ be its Szegö kernel. Let $f$ be a function from $E$ to $\bar{\Omega}$ such that the kernel $K(z, \zeta)\left[S_{\Omega}(f(z), f(\zeta))\right]^{-1}$ is positive-definite on $E$. Then there is a unique holomorphic function $F$ on $D$ with $\left.F\right|_{E}=f$ and such that the kernel $K(z, \zeta)\left[S_{\Omega}(F(z), F(\zeta))\right]^{-1}$ is positive-definite on $D$. Moreover, either $F(z) \in \Omega$ for every $z \in D$ or $F(z) \equiv c$ for every $z \in D$, where $c$ is a constant lying on $\partial \Omega$.

Proof. As in the previous proof, we let $\phi$ be a conformal mapping of $\Omega$ onto $\Delta$, which now extends to a $C^{1}$-diffeomorphism of $\bar{\Omega}$ onto $\bar{\Delta}$. We may therefore define $f_{1}=\phi \circ f$, so that $\left|f_{1}(z)\right| \leqslant 1$ for all $z \in E$. Identity (3.3) is now valid in view of (3.1), (3.2) and (3.4). In particular, the kernel $K(z, \zeta)\left(1-f_{1}(z) \overline{f_{1}(\zeta)}\right)$ is positive-definite on $E$. Arguing as before we deduce the existence of a unique holomorphic function $F_{1}$ on $D$ with $\left.F_{1}\right|_{E}=f_{1}$ and $\left|F_{1}(z)\right| \leqslant 1$ for all $z \in D$. By the maximum modulus principle either $\left|F_{1}(z)\right|<1$ for all $z \in D$ or $F_{1}(z) \equiv c_{1}$ for all $z \in D$, where $c_{1}$ is a constant with $\left|c_{1}\right|=1$. Set, again, $F=\phi^{-1} \circ F_{1}$. Then $\left.F\right|_{E}=f$ and either $F(z) \in \Omega$ for all $z \in D$ or $F(z) \equiv \phi^{-1}\left(c_{1}\right) \in \partial \Omega$ for all $z \in D$, in which case $f$ was originally a constant $c=\phi^{-1}\left(c_{1}\right)$. Clearly, $F$ is holomorphic on $D$ in both cases. Now, in the case that $F(z) \equiv c \in \partial \Omega$, we have trivially that $\left[S_{\Omega}(F(z), F(\zeta))\right]^{-1} \equiv 0$, for all $z, \zeta \in D$, while in the case that $F(z) \in \Omega$ for $z \in D$, the previous proof of Corollary 3.8 applies. We conclude that $K(z, \zeta)\left[S_{\Omega}(F(z), F(\zeta))\right]^{-1}$ is a positive-definite kernel on $D$, and the proof is complete.

The classical interpolation theorem of Pick [24] and Nevanlinna [23] characterizes restrictions of bounded holomorphic functions on the unit disk $\Delta$ in terms of the Szegö kernel $S_{\lrcorner}(z, \zeta)=\{2 \pi(1-z \bar{\zeta})\}^{-1}$. Thus, if $E$ is an arbitrary subset of $\Delta$ and if 
$f$ is a function on $E$, then $f$ is a restriction of a holomorphic function $F$ on $\Delta$ with $\|F\|_{\infty} \leqslant 1$ if and only if the kernel $S_{\Delta}(z, \zeta)(1-f(z) \overline{f(\zeta)})$ is positive-definite on $E$. A simple conformal mapping argument (see Corollary 3.9 ) shows that the same is true if $\Delta$ is replaced by any hyperbolic simply connected plane domain $\Omega$. In the case that $E$ is a set of uniqueness for $\mathcal{O}(\Omega)$, the above theorem becomes a special case of our Corollary 3.7, however if $E$ is not a set of uniqueness for $\mathcal{O}(\Omega)$ the result cannot be proved by our methods. In fact, in this degree of generality, the hypothesis that $E$ is a set of uniqueness for $\mathcal{O}(\Omega)$ is essential. Korányi and Pukansky [19] have given an example of a set $E$ consisting of two points in the unit polydisk $\Delta^{n}$ in $\mathbf{C}^{n}(n \geqslant 2)$ such that the conclusion of Theorem 3.5 does not hold when $K$ is the Szegö kernel for $\Delta^{n}$. Minor modifications of this example show that Theorem 3.5 cannot hold for arbitrary sets $E$ if $K$ is a kernel on the unit ball $B_{n}$ in $\mathbf{C}^{n}$ of the form $K_{\alpha}(z, \zeta)$ $=(1-\langle z, \zeta\rangle)^{-\alpha}$ with $\alpha>1$. More specifically, the following proposition holds.

3.10. Proposition. The kernel $K_{\alpha}(z, \zeta)=(1-z \bar{\zeta})^{-\alpha}$ is sesqui-holomorphic and positive-definite on the unit disk $\Delta$ for any $\alpha \in(0, \infty)$. Moreover, let $f$ be an arbitrary function on an arbitrary subset $E$ of $\Delta$, and consider the following statements:

(i) There is an $F \in H^{\infty}(\Delta)$ with $\|F\|_{\infty} \leqslant 1$ and $\left.F\right|_{E}=f$.

(ii) The kernel $K_{\alpha}(z, \zeta)(1-f(z) \overline{f(\zeta)})$ is positive-definite on $E$.

Then, the implication (i) $\Rightarrow$ (ii) is always true if and only if $\alpha \geqslant 1$, while the implication (ii) $\Rightarrow$ (i) is always true if and only if $0<\alpha<1$.

Proof. The first part of the proposition follows from the fact that $K_{\alpha}(z, \zeta)=$ $(1-z \bar{\zeta})^{-\alpha}, \alpha>0$, is the reproducing kernel of the functional Hilbert space

$$
H_{\alpha}(\Delta)=\left\{g \in \mathcal{O}(\Delta):\|g\|_{\alpha}<\infty\right\}
$$

where

$$
\|g\|_{\alpha}^{2}=\Gamma(\alpha) \sum_{m=0}^{\infty} \frac{m !}{\Gamma(\alpha+m)}\left|b_{m}\right|^{2} ; \quad g(z)=\sum_{m=0}^{\infty} b_{m} z^{m}, \quad z \in \Delta .
$$

As for the second part, we argue as follows: If $\alpha>1$, then $H_{\alpha}(\Delta)$ is the weighted Bergman space $A_{\phi_{\alpha}}^{2}(\Delta)$ with the weight $\phi_{\alpha}(z)=\pi^{-1}(\alpha-1)\left(1-|z|^{2}\right)^{\alpha-2}, z \in \Delta$. If $\alpha=1$, then $H_{1}(\Delta)$ is the Hardy space $H^{2}(\Delta)$ with the norm scaled by the factor $1 / \sqrt{2 \pi}$. It follows that $K_{\alpha}$ possesses the contraction-property when $\alpha \geqslant 1$, and thus, by Theorem 2.12 or Corollary 3.7, (i) $\Rightarrow$ (ii), if $\alpha>1$. If $0<\alpha<1$, we let $E=\Delta$ and choose $f$ as

$$
f(z)=z \frac{z-r}{1-r z}, \quad z \in \Delta
$$

with

$$
r=\frac{\sqrt{1-s}-\sqrt{(1-s-\varepsilon) /(1-s)}}{1-\sqrt{1-s-\varepsilon}},
$$

where $s$ and $\varepsilon$ are constants satisfying

$$
0<s<1, \quad 0<\varepsilon<s^{\alpha}-s \quad(0<\alpha<1) .
$$


Thus $f=F \in H^{\infty}(\Delta)$ with $\|f\|_{\infty} \leqslant 1$, so that (i) is satisfied. However, (ii) is not satisfied. Indeed, let $z_{1}=0, z_{2}=\sqrt{1-s}$; thus $z_{1}, z_{2} \in \Delta$ and $f\left(z_{1}\right), f\left(z_{2}\right) \in \Delta$ with $f\left(z_{1}\right)=0$ and $f\left(z_{2}\right)=\sqrt{1-s-\varepsilon}$. It follows that

$$
\operatorname{det}\left[K_{\alpha}\left(z_{i}, z_{j}\right)\left(1-f\left(z_{i}\right) \overline{f\left(z_{j}\right)}\right)\right]_{i, j=1}^{2}=(s+\varepsilon) s^{-\alpha}-1<0
$$

and thus (ii) is violated. We now prove the assertion concerning the implication (ii) $\Rightarrow$ (i). For $\alpha \in(0,1]$, we have

$$
K_{1}(z, \zeta)(1-f(z) \overline{f(\zeta)})=K_{1-\alpha}(z, \zeta) K_{\alpha}(z, \zeta)(1-f(z) \overline{f(\zeta)})
$$

In particular, if (ii) is satisfied then, since $K_{1-\alpha}$ is positive-definite on $\Delta$ by the first part of the proposition, $K_{1}(z, \zeta)(1-f(z) \overline{f(\zeta)})$ is positive-definite on $E$ as a product of two positive-definite kernels on $E$ (here we have used Schur's theorem [11, p. 9]). Since $K_{1}=2 \pi S_{\Delta}$, we have that $S_{\Delta}(z, \zeta)(1-f(z) \overline{f(\zeta)})$ is positive-definite on $E$, and thus (i) follows from the previously mentioned Pick-Nevanlinna interpolation theorem. Thus (ii) $\Rightarrow$ (i) if $0<\alpha \leqslant 1$. If $\alpha>1$, we let $E=\left\{z_{1}, z_{2}\right\}$ be a set consisting of two points in $\Delta$, given by $z_{1}=0$ and $z_{2}=\sqrt{1-s}$ with $0<s<1$. We choose $f$ to be a function on $E$, specified by $f\left(z_{1}\right)=0$ and $f\left(z_{2}\right)=\sqrt{1-s+\varepsilon}$, where $\varepsilon$ is a constant satisfying

$$
0<\varepsilon<s-s^{\alpha} \quad(0<s<1, \alpha>1) .
$$

It follows that

$$
\operatorname{det}\left[K_{\alpha}\left(z_{i}, z_{j}\right)\left(1-f\left(z_{i}\right) f\left(z_{j}\right)\right)\right]_{i, j=1}^{2}=(s-\varepsilon) s^{-\alpha}-1>0
$$

and thus (ii) is satisfied. However, (i) is not satisfied. In fact, if (ii) is satisfied, then, since $F(0)=f(0)=0$ and $\left|F\left(z_{2}\right)\right|=\left|f\left(z_{2}\right)\right| \neq\left|z_{2}\right|$, we must have $|F(z)|<|z|$ for all $z \in \Delta \backslash\{0\}$ by Schwarz's lemma. But this is violated by virtue of $\left|F\left(z_{2}\right)\right|=\left|f\left(z_{2}\right)\right|=$ $\sqrt{1-s+\varepsilon}>\sqrt{1-s}=\left|z_{2}\right|$, and the proof is complete.

The last proposition shows that the interpolation theorem for arbitrary subsets of $\Delta$ seems to depend heavily on the close connection between the Szegö kernel function and the automorphism group of $\Delta$, which we do not have in general. It would be of interest to know whether there are kernels on other homogeneous domains, the ball for example, for which the analogue of the classical theorem holds for arbitrary sets $E$. A very partial result in this direction is the following corollary.

3.11. Corollary. Let $D$ be a domain in $\mathbf{C}^{n}$ and for $j=1,2$ let $\Omega_{j}$ be any hyperbolic simply connected plane domain with the Szegö kernel $S_{\Omega_{j}}$. Let $G$ be a holomorphic function of $D$ into $\Omega_{2}$ and let $E$ be an arbitrary subset of $D$. Let $f$ be a function from $E$ into $\Omega_{1}$ such that, for some $0<\alpha \leqslant 1$, the kernel $\left[S_{\Omega_{2}}(G(z), G(\zeta))\right]^{\alpha}\left[S_{\Omega_{1}}(f(z), f(\zeta))\right]^{-1}$ is positive-definite on $E$. Then there exists a holomorphic function $F$ on $D$ such that $\left.F\right|_{E}=f$ and $F(z) \in \Omega_{1}$ for all $z \in D$. If, in addition, $\partial \Omega_{1}$ is of class $C^{1}$ with a Dini continuous normal, then in the assumptions on $f, \Omega_{1}$ may be replaced by $\bar{\Omega}_{1}$ while in the conlusions on $F, F(z) \in \Omega_{1}$ for all $z \in D$ is replaced by either $F(z) \in \Omega_{1}$ for all $z \in D$ of $F(z) \equiv c$ for every $z \in D$, where $c$ is a constant lying on $\partial \Omega$. 
Proof. Let $\phi_{j}$ be a conformal mapping of $\Omega_{j}$ onto the unit disk $\Delta(j=1,2)$. Define $f_{1}=\phi_{1} \circ f$ and $G_{2}=\phi_{2} \circ G$. Using (3.1)-(3.2) and the notation of the last proposition, we obtain

$$
\begin{aligned}
K_{\alpha}\left(G_{2}(z), G_{2}(\zeta)\right)\left(1-f_{1}(z) \overline{f_{1}(\zeta)}\right) \\
=(2 \pi)^{\alpha-1}\left\{\frac{\phi_{1}^{\prime}(f(z))}{\phi_{2}^{\prime}(G(z))^{\alpha}}\right\}^{1 / 2}\left\{\overline{\frac{\phi_{1}^{\prime}(f(\zeta))}{\phi_{2}^{\prime}(G(\zeta))^{\alpha}}}\right\}^{1 / 2} \\
\quad \times\left[S_{\Omega_{2}}(G(z), G(\zeta))\right]^{\alpha}\left[S_{\Omega_{1}}(f(z), f(\zeta))\right]^{-1}
\end{aligned}
$$

and thus $K_{\alpha}\left(G_{2}(z), G_{2}(\zeta)\right)\left(1-f_{1}(z) \overline{f_{1}(\zeta)}\right)$ is positive-definite on $E$. On $G_{2}(E)$, which is a subset of $\Delta$, we define the function $f_{0}$ by $f_{0}\left(G_{2}(z)\right)=f_{1}(z)$ for all $z \in E$. Letting $\omega=G_{2}(z), \tau=G_{2}(\zeta)$ we see that $K_{\alpha}(\omega, \tau)\left(1-f_{0}(\omega) \overline{f_{0}(\tau)}\right)$ is positive-definite on $G_{2}(E)$. It follows from Proposition 3.10, since $0<\alpha \leqslant 1$, that there is an $F_{0} \in H^{\infty}(\Delta)$ with $\left\|F_{0}\right\| \leqslant 1$ and $\left.F_{0}\right|_{G_{2}(E)}=f_{0}$. Since $\left|f_{0}\left(G_{2}(z)\right)\right|=\left|f_{1}(z)\right|=$ $\left|\phi_{1}(f(z))\right|<1$ we conclude, by the maximum modulus principle, that $F_{0}(z) \in \Delta$ for $z \in G_{2}(E)$. We now define $F$ by

$$
F=\phi_{1}^{-1} \circ F_{0} \circ \phi_{2} \circ G
$$

Then $F \in \mathcal{O}(D),\left.F\right|_{E}=f$ and $F(z) \in \Omega_{1}$ for all $z \in \Delta$. The additional assertion connected with the smoothness of $\partial \Omega_{1}$ may be proved in a similar manner by appealing to arguments of the proof of Corollary 3.9. The proof is now complete.

4. Interpolation from a subset of the boundary. In this section we obtain analogues of the results of the previous section when the set $E$ is a subset of the boundary. For this purpose it is of course sufficient to establish the analogues of Theorems 3.4 and 3.5. These analogues are formulated in terms of the Hardy space of the domain $D$. To a certain extent the definition of the Hardy spaces is similar to that of the Bergman spaces except that the integration is now taken on the boundary (or on the distinguished boundary) of $D$. It is not our purpose here to give a detailed presentation of the very delicate ideas involved in these spaces. Instead, our aim is to show how these spaces tie up with the present theory. For this reason, and, in order to avoid unnecessary difficulties, we now assume that $D$ is a bounded smooth domain in $\mathbf{C}^{n}$. The boundary $\partial D$ is taken to be of class $C^{2}$, even though, for example, the class $C^{1, \varepsilon}(0<\varepsilon<1)$ would suffice. In fact, when $n=1$ the class of allowable domains $D$ includes any plane domain whose boundary is rectifiable.

Throughout this section $D$ will denote a bounded domain in $\mathbf{C}^{n}$ with a $C^{2}$ boundary, and $r$ will denote some fixed characterizing-function for $D$, i.e. $r$ is a real-valued $C^{2}$ function on $\mathbf{C}^{n}$ such that $D=\left\{z \in \mathbf{C}^{n}: r(z)<0\right\}$ and $|\nabla r(z)|>0$ for $z \in \partial D$. There exists an $\varepsilon_{0}>0$ such that the domains $D_{\varepsilon}=\left\{z \in \mathbf{C}^{n}: r(z)<-\varepsilon\right\}$ are relatively compact subdomains of $D$ with $C^{2}$ boundaries for all $\varepsilon \in\left(0, \varepsilon_{0}\right)$. Let $\sigma$ and $\sigma_{\varepsilon}$ denote the surface measures on $\partial D$ and $\partial D_{\varepsilon}$, respectively.

For $0<p<\infty$, the Hardy class $H^{p}(D)$ is defined as the space of all $f$ in $\mathcal{O}(D)$ with

$$
\|f\|_{p}=\varlimsup_{\varepsilon \rightarrow 0^{+}}\left\{\int_{\partial D_{\varepsilon}}|f|^{p} d \sigma_{\varepsilon}\right\}^{1 / p}<\infty .
$$


If $|f|^{p}$ is replaced by $\log ^{+}|f|=\max \{\log |f|, 0\}$, we obtain the Nevanlinna class $H^{0}(D)$. The fact that $\log |f|$ and $|f|^{p}$ are plurisubharmonic ensures that this definition of the classes $H^{p}(D), 0 \leqslant p<\infty$, is independent of the particular choice of the characterizing-function $r$. If $\|f\|_{p}$ is replaced by $\|f\|_{\infty}=\sup \{|f(z)|: z \in D\}$, we obtain the customary space $H^{\infty}(D)$. It follows that $H^{q}(D) \subset H^{p}(D)$ whenever $0 \leqslant p \leqslant q \leqslant \infty$. Moreover, for $1 \leqslant p \leqslant \infty,\|\cdot\|_{p}$ is a norm which makes $H^{p}(D)$ into a Banach space.

Any function $f \in H^{0}(D)$ has nontangential limits at almost every point of $\partial D$. We denote by $f^{*}$ the boundary value function of $f$. It follows that $\log ^{+}\left|f^{*}\right| \in L_{1}(\partial D)$ and, moreover, if $f \in H^{p}(D)$ then $f^{*} \in L^{p}(\partial D), 0<p \leqslant \infty$. The function $f^{*}$ can be realized as a norm limit of "dilations" of $f$ in the following sense. Let $\pi$ be the normal projection of a tubular neighborhood $T$ of $\partial D$ onto $\partial D$. Then for $\varepsilon>0$ sufficiently small, $\pi_{\varepsilon}=\left.\pi\right|_{\partial D_{\varepsilon}}$ is a $C^{1}$-diffeomorphism of $\partial D_{\varepsilon}$ onto $\partial D$ and we define $u_{\varepsilon}=u \circ \pi_{\varepsilon}^{-1}$ for any function $u$ on $D$. It follows that $f_{\varepsilon}$ is continuous on $\partial D$ for any $f \in H^{0}(D)$. Moreover, if also $f \in H^{p}(D), 0<p<\infty$ then $f_{\varepsilon}$ converges to $f^{*}$ in $L^{p}(\partial D)$. For $0<p \leqslant \infty, H^{p}(\partial D)=\left\{f^{*} \in L^{p}(\partial D): f \in H^{p}(D)\right\}$ is a closed subspace of $L^{p}(\partial D)$, and for the correspondence $f \rightarrow f^{*}$ is a linear isometry of $H^{p}(D)$ onto $H^{p}(\partial D)$. It follows that for $0<p \leqslant \infty, H^{p}(D)$ can be identified with the closed subspace $H^{p}(\partial D)$ of $L^{p}(\partial D)$ by identifying $f \in H^{p}(D)$ with its nontangential boundary value function $f^{*} \in H^{p}(\partial D)$. In what follows, we make this identification implicitly. We refer the reader to Stein [29, pp. 32-52] for further details on boundary behavior of holomorphic functions.

The Hardy space $H^{2}(D)$ may therefore be regarded as a functional Hilbert space of holomorphic functions on $D$ with square integrable boundary values. Its reproducing kernel $S=S(z, \zeta)$ is the Szegö kernel of $D$. The Szegö kernel represents the orthogonal projection $\mathbf{S}$ of $L^{2}(\partial D)$ onto $H^{2}(\partial D)$ in the sense that if $\rho \in L^{2}(\partial D)$ then $\mathbf{S} \rho \in H^{2}(\partial D)$ is the boundary value function of the holomorphic function

$$
\{\mathbf{S} \rho\}(z)=\langle\rho, S(\cdot, z)\rangle=\int_{\partial D} \rho(\zeta) \overline{S(\zeta, z)} d \sigma(\zeta), \quad z \in D,
$$

which is also a member of $H^{2}(D)$.

Before proceeding we make a brief remark concerning notation. In what follows we will occasionally use iterated integrals of the form

$$
\int_{\partial D} \int_{\partial D} L(z, \zeta) \rho(z) \overline{\rho(\zeta)} d \sigma(\zeta) d \sigma(z)
$$

where $L$ is a kernel subordinate to the Szegö kernel and $\rho \in H^{2}(\partial D)$. If $L(z, \zeta)$ has singularities in $\partial D \times \partial D$, some care must be taken in the interpretation of (4.1). If $L$ is singular in $\partial D \times \partial D$, then (4.1) is to be interpreted to mean

$$
\lim _{\varepsilon \rightarrow 0^{+}} \int_{\partial D} \int_{\partial D} L(z-\varepsilon \nu(z), \zeta) \rho(z) \overline{\rho(\zeta)} d \sigma(\zeta) d \sigma(z)
$$

where $\nu(z)$ denotes the outward unit normal vector to $\partial D$ at the point $z \in \partial D$.

It should be noted that in (4.1) and (4.2) the integrations may be performed over a subset of $\partial D$ by means of suitable characteristic functions. 
Throughout this section, $E$ will be a Borel set in $\partial D$ with positive surface measure, i.e. $\sigma(E)>0$. The following lemma says, loosely speaking, that a subset of the boundary with positive measure is a set of uniqueness for the Nevanlinna class. For a proof, we refer to Beatrous [2].

4.1. LEMMA. Let $f \in H^{0}(D)$ and assume that $f$ has nontangential limit 0 at almost every point of $E$. Then $f \equiv 0$ on $D$.

For any function $\rho$ on $E$, we denote by $Q_{E} \rho$ the function on $\partial D$ which agrees with $\rho$ on $E$ and which vanishes on $\partial D \backslash E$.

4.2. Corollary. The vector space $\mathscr{H}_{0}=\left\{\mathbf{S} Q_{E} \bar{\rho}: \rho \in L^{\infty}(E)\right\}$ is a dense subspace of $H^{2}(\partial D)$.

Proof. Let $f \in H^{2}(\partial D)$ with $f \perp \mathscr{H}_{0}$. Then for $\rho \in L^{\infty}(E)$, we have

$$
0=\left\langle f, \mathbf{S} Q_{E} \bar{\rho}\right\rangle=\left\langle f, Q_{E} \bar{\rho}\right\rangle=\int_{E} f \rho d \sigma .
$$

Hence $f$ vanishes on $E$, and thus by Lemma 4.1 we have $f \equiv 0$ on $D$.

We now turn to the boundary analogue of Theorem 3.4 .

4.3. THEOREM. Let L be a kernel on D which is subordinate to the Szegö kernel and let $f$ be a measurable function on $E$ such that, for some nonnegative constant $c$,

$$
\int_{E} \int_{E}\left(c^{2} L(z, \zeta)-f(z) \overline{f(\zeta)}\right) \rho(z) \overline{\rho(\zeta)} d \sigma(\zeta) d \sigma(z) \geqslant 0
$$

for all $\rho \in L^{\infty}(E)$. Then there is a unique $F \in H^{2}(D)$ with $\left.F\right|_{E}=f$ (in the sense that $F(z)$ tends to $f(\zeta)$ as $z$ tends nontangentially to $\zeta$ for almost every $\zeta \in E)$. Moreover, the kernel $c^{2} L(z, \zeta)-F(z) \overline{F(\zeta)}$ is positive-definite on $D$. In particular, $\|F\| \leqslant c \sqrt{\|L\|}$.

Proof. Let $T$ be the operator in $\mathscr{B}\left(H^{2}(D)\right)$ which is represented by the kernel $L$. Now, condition (4.3), whose interpretation is by means of (4.1)-(4.2), is clearly equivalent to the inequality

$$
c^{2} \int_{E} \int_{E} L(z, \zeta) \rho(z) \overline{\rho(\zeta)} d \sigma(\zeta) d \sigma(z) \geqslant\left|\int_{E} f \rho d \sigma\right|^{2} .
$$

But the left-hand side is equal to $c^{2}\left\langle T^{*} \mathbf{S} Q_{E} \bar{\rho}, \mathbf{S} Q_{E} \bar{\rho}\right\rangle$. Thus, since $\left\|T^{*}\right\|=\|L\|$, (4.3) implies that for $\rho \in L^{\infty}(E)$ we have

$$
\left|\int_{E} \bar{\rho} \bar{f} d \sigma\right|^{2} \leqslant c^{2}\|L\| \cdot\left\|\mathbf{S} Q_{E} \bar{\rho}\right\|^{2} .
$$

Thus, in view of Corollary 4.2, there exists a unique linear functional $l$ on $H^{2}(D)$ with $\|l\| \leqslant c \sqrt{\|L\|}$ such that $l\left(\mathbf{S} Q_{E} \bar{\rho}\right)=\int_{E} \bar{\rho} \bar{f} d \sigma$ for all $\rho \in L^{\infty}(E)$. Let $F \in H^{2}(D)$ be the function which represents the linear functional $l$. Then for $\rho \in L^{\infty}(E)$ we have

$$
\int_{E} \bar{\rho} \bar{f} d \sigma=l\left(\mathbf{S} Q_{E} \overline{\boldsymbol{\rho}}\right)=\left\langle\mathbf{S} Q_{E} \bar{\rho}, F\right\rangle=\left\langle Q_{E} \bar{\rho}, \mathbf{S} F\right\rangle=\left\langle Q_{E} \overline{\boldsymbol{\rho}}, F\right\rangle=\int_{E} \overline{\boldsymbol{\rho}} \bar{F} d \sigma
$$


and thus $F$ agrees with $f$ at almost every point of $E$. Note also that

$$
\|F\|=\|l\| \leqslant c \sqrt{\|L\|} .
$$

To show that the kernel $c^{2} L(z, \zeta)-F(z) \overline{F(\zeta)}$ is positive-definite on $D$, we show that it corresponds to a positive operator on $H^{2}(D)$. Let $T_{F}$ be the operator induced by the kernel $F(z) \overline{F(\zeta)}$; thus the operator in question is $c^{2} T-T_{F}$. To verify that $c^{2} T-T_{F}$ is positive on $H^{2}(D)$, it suffices to show that it is positive on $\mathscr{H}_{0}=\left\{\mathbf{S} Q_{E} \bar{\rho}\right.$ : $\left.\rho \in L^{2}(E)\right\}$ which by Corollary 4.2 is a dense subspace of $H^{2}(D)$. But $\left\langle\left(c^{2} T-T_{F}\right) \mathbf{S} Q_{E} \bar{\rho}, \mathbf{S} Q_{E} \bar{\rho}\right\rangle$ is precisely the left-hand side of (4.3) which is nonnegative by hypothesis. Finally, $\|F\| \leqslant c \sqrt{\|L\|}$ has already been derived in (4.4); it could also have been established, since $c^{2} L(z, \zeta)-F(z) \overline{F(\zeta)}$ is positive-definite on $D$, as a particular case of Corollary 2.10. This concludes the proof.

Specializing the preceding theorem to the Szegö kernel yields a local characterization of the boundary values of functions in $H^{2}(D)$.

4.4. Corollary. Let $f$ be a measurable function on $E$ and let $c$ be a nonnegative constant. Then the following conditions are equivalent:

(i) There is a unique function $F \in H^{2}(D)$ with $\left.F\right|_{E}=f$ and $\|F\| \leqslant c$.

(ii) There is a unique function $F \in H^{2}(D)$ with $\left.F\right|_{E}=f$ and such that the kernel $c^{2} S(z, \zeta)-F(z) \overline{F(\zeta)}$ is positive-definite on $D$.

(iii) For every $\rho \in L^{\infty}(E)$, we have

$$
\int_{E} \int_{E}\left(c^{2} S(z, \zeta)-f(z) \overline{f(\zeta)}\right) \rho(z) \overline{\rho(\zeta)} d \sigma(\zeta) d \sigma(z) \geqslant 0
$$

Proof. The implication (i) $\Rightarrow$ (ii) is a special case of Theorem 2.2. As for the implication (ii) $\Rightarrow$ (iii), we let $I$ be the identity operator on $H^{2}(D)$ and let $T_{F}$ be the operator on $H^{2}(D)$ which is represented by the kernel $F(z) \overline{F(\zeta)}$. It follows that $c^{2} I-T_{F}$ is a positive operator on $H^{2}(D)$. In particular, for any $\rho \in L^{\infty}(E)$ we have $\left\langle\left(c^{2} I-T_{F}\right) \mathbf{S} Q_{E} \bar{\rho}, \mathbf{S} Q_{E} \bar{\rho}\right\rangle \geqslant 0$ and (iii) follows. Finally, the implication (iii) $\Rightarrow$ (i) is a special case of Theorem 4.3, since $\|\mathbf{S}\|=1$. This concludes the proof.

By taking $f \equiv 0$ in Theorem 4.3 we obtain a result on the persistence of positivedefiniteness.

4.5. Corollary. Let $L$ be a kernel on $D$ which is subordinate to the Szegö kernel, and suppose that for every $\rho \in L^{\infty}(E)$ we have

$$
\int_{E} \int_{E} L(z, \zeta) \rho(z) \overline{\rho(\zeta)} d \sigma(\zeta) d \sigma(z) \geqslant 0 .
$$

Then $L$ is positive-definite on $D$.

Our next result is the boundary analogue of Theorem 3.5. For the unit disk, the implication (iv) $\Rightarrow$ (i) is due to Rosenblum and Rovnyak [25] and FitzGerald [15]. The general case is due to Beatrous [2] under the more restrictive hypothesis that $E$ contains a relatively open subset of $\partial D$. For the other implications, see also Burbea $[4]$, and Burbea and Masani $[9,10]$. 
4.6. THEOREM. Let $f$ be a measurable function on $E$ and let $c$ be a nonnegative constant. Then the following conditions are equivalent:

(i) There is a unique function $F \in H^{\infty}(D)$ with $\left.F\right|_{E}=f$ and $\|F\|_{\infty} \leqslant c$.

(ii) There is a unique function $F \in H^{\infty}(D)$ with $\left.F\right|_{E}=f$ and $S(z, \zeta)\left(c^{2}-\right.$ $F(z) \overline{F(\zeta)})$ is positive-definite on $D$.

(iii) There is a unique function $F \in H^{\infty}(D)$ with $\left.F\right|_{E}=f$ and $M_{F}$, the multiplication-operator due to $F$ on $H^{2}(D)$, satisfies $\left\|M_{F}\right\| \leqslant c$. In this case $M_{F}^{*}=\mathbf{S} M_{\bar{F}}$ with $\left\|M_{\bar{F}}\right\|=\left\|M_{F}\right\|=\|F\|_{\infty} \leqslant c$.

(iv) For every $\rho \in L^{\infty}(E)$, we have

$$
\int_{E} \int_{E} S(z, \zeta)\left(c^{2}-f(z) \overline{f(\zeta)}\right) \rho(z) \overline{\rho(\zeta)} d \sigma(z) d \sigma(\zeta) \geqslant 0 .
$$

Proof. Since the Szegö kernel has the contraction-property, the implications (i) $\Rightarrow$ (ii) $\Rightarrow$ (iii) follow easily from Theorem 2.11 (see also the proof of Corollary 3.7). As for the implication (iii) $\Rightarrow$ (iv), we observe that (iii) implies that $c^{2} I-$ $M_{F} \mathbf{S} M_{\bar{F}}$ is a positive operator on $H^{2}(D)$. In particular, in view of Corollary 4.2 , for every $\rho \in L^{\infty}(E)$ we have $\left\langle\left(c^{2} I-M_{F} S M_{\bar{F}}\right) S Q_{E} \bar{\rho}, S Q_{E} \bar{\rho}\right\rangle \geqslant 0$, and (iv) follows. Condition (iv) is thus interpreted as in (4.1)-(4.2). We consider $S(z-\varepsilon \nu(z), \zeta)$ and then take $\lim \varepsilon \rightarrow 0^{+}$.

We now prove the implication (iv) $\Rightarrow$ (i). Let $g$ be any function in $H^{2}(D)$. By Theorem 2.2, the kernel $S_{g}(z, \zeta)=\|g\|^{2} S(z, \zeta)-g(z) \overline{g(\zeta)}$ is positive-definite on $D$. Thus, for $\rho \in L^{\infty}(E)$ we have

$$
\begin{aligned}
\left|\int_{E} f g \rho d \sigma\right|^{2} & \leqslant\left|\int_{E} f g \rho d \sigma\right|^{2}+\int_{E} \int_{E} S_{g}(z, \zeta) f(z) \overline{f(\zeta)} \rho(z) \overline{\rho(\zeta)} d \sigma(\zeta) d \sigma(z) \\
& =\|g\|^{2} \int_{E} \int_{E} S(z, \zeta) f(z) \overline{f(\zeta)} \rho(z) \overline{\rho(\zeta)} d \sigma(\zeta) d \sigma(z) \\
& \leqslant c^{2}\|g\|^{2} \int_{E} \int_{E} S(z, \zeta) \rho(z) \overline{\rho(\zeta)} d \sigma(\zeta) d \sigma(z)
\end{aligned}
$$

Thus it follows from Corollary 4.4. that there is a function $M(g) \in H^{2}(D)$ with $\left.M(g)\right|_{E}=f g$ and $\|M(g)\| \leqslant c\|g\|$. In particular, this is true for the constant function 1 which is in $H^{2}(D)$, because $D$ is bounded. Set $F=M(1)$. Then $F \in H^{2}(D)$ and $\left.F\right|_{E}=f$ and $\|F\| \leqslant c\|1\|=c \sqrt{\sigma(\partial D)}$. To complete the proof, we must show that in fact $\|F\|_{\infty} \leqslant c$. For $g \in H^{2}(D)$, the function $F g$ is clearly in $H^{1}(D)$ and it agrees with $M(g)$ on $E$. Thus it follows from Lemma 4.1 that $M(g)=F g$ for every $g \in H^{2}(D)$. We therefore deduce that $F$ has the multiplication-property with respect to the Szegö kernel $S$. In particular, $M_{F}(g)=M(g)=F g$ with $\left\|M_{F}(g)\right\| \leqslant c\|g\|$, for all $g \in H^{2}(D)$. It follows from Proposition 3.6 that $F \in H^{\infty}(D)$ and $\|F\|_{\infty}=\left\|M_{F}\right\|$ $\leqslant c$. The proof is now complete.

Condition (iv) of Theorem 4.6 can often be formulated in terms of certain principal value integrals on $\partial D$. For example, if $D$ is the unit ball $B_{n}$ of $\mathbf{C}^{n}$ then, for $\rho \in L^{2}\left(\partial B_{n}\right)$, we have

$$
\{\mathbf{S} \rho\}(z)=\frac{(n-1) !}{2 \pi^{n}} \lim _{\varepsilon \rightarrow 0^{+}} \int_{|1-\langle z, \zeta\rangle|>\varepsilon}(1-\langle z, \zeta\rangle)^{-n} \rho(\zeta) d \sigma(\zeta)+\frac{1}{2} \rho(z)
$$


for almost all $z \in \partial B_{n}$ (see Korányi and Vági [20]). It follows that condition (iv) of Theorem 4.6 is equivalent to

$$
\begin{gathered}
\frac{(n-1) !}{2 \pi^{n}} \lim _{\varepsilon \rightarrow 0^{+}} \iint_{(E \times E)_{\varepsilon}} \frac{1-f(z) \overline{f(\zeta)}}{(1-\langle z, \zeta\rangle)^{n}} \rho(z) \overline{\rho(\zeta)} d \sigma(z) d \sigma(\zeta) \\
+\frac{1}{2} \int_{E}\left(1-|f|^{2}\right)|\rho|^{2} d \sigma \geqslant 0,
\end{gathered}
$$

for all $\rho \in L^{\infty}(E)$, where $E$ is a Borel subset of $\partial B_{n}$ and

$$
(E \times E)_{\varepsilon}=\{(z, \zeta) \in E \times E:|1-\langle z, \zeta\rangle|>\varepsilon\} .
$$

In particular, if the measurable function $f$ on $E$ satisfies $|f|=1$ almost everywhere on $E$, then the second integral in (4.5) vanishes, and thus (4.5) is equivalent to

$$
\lim _{\varepsilon \rightarrow 0^{+}} \iint_{(E \times E)_{\varepsilon}} \frac{1-f(z) \overline{f(\zeta)}}{(1-\langle z, \zeta\rangle)^{n}} \rho(z) \overline{\rho(\zeta)} d \sigma(z) d \sigma(\zeta) \geqslant 0 \quad(|f|=1 \text { a.e. on } E) \text {. }
$$

An interesting application involving condition (4.6) occurs when $n=1$, i.e. when $B_{n}$ is the unit disk $\Delta$. Note that if $E$ is an arc on the unit circle $\partial \Delta$ and if $f \in C^{1}(E)$ with $|f|=1$ on $E$, then the integral in (4.6) is nonsingular. In this case, (4.6) becomes

$$
\int_{E} \int_{E} \frac{1-f(z) \overline{f(\zeta)}}{1-z \bar{\zeta}} \rho(z) \overline{\rho(\zeta)} d \sigma(z) d \sigma(\zeta) \geqslant 0, \quad \rho \in L^{\infty}(E)
$$

We can also give a discrete version of condition (4.7). For $f$ as above, define the kernel $K_{f}$ on $E \times E$ by

$$
K_{f}(z, \zeta)= \begin{cases}\frac{1-f(z) \overline{f(\zeta)}}{1-z \bar{\zeta}}, & z \neq \zeta \\ -i \frac{d}{d \Theta} \log f(\zeta), & z=\zeta\left(=e^{i \Theta}\right)\end{cases}
$$

Then (4.7) is equivalent to the assertion that the kernel $K_{f}$ is positive-definite on $E$ (see also FitzGerald [15]). Thus, an elementary conformal mapping argument shows that Theorem 4.6 implies the classical theorem of Loewner [21] (see also Donoghue $[11, \mathrm{pp} .85,96,126])$ giving necessary and sufficient conditions for a real-valued $C^{1}$ function on an interval of the real line to be the boundary value of a holomorphic function from the upper half-plane into itself.

Similar considerations apply to an arbitrary bounded domain $D$ in $\mathbf{C}$ whose boundary $\partial D$ is of class $C^{1}$ with a Dini continuous normal. In this case, using the fact that the singularity of the Szegö kernel $S_{D}(z, \zeta)$ is essentially the same as that of the Cauchy kernel (see Bergman [3, pp. 110-120] and Burbea [5]), we see that condition (iv) of Theorem 4.6 is equivalent to 


$$
\begin{gathered}
\lim _{\varepsilon \rightarrow 0^{+}} \iint_{(E \times E)_{\varepsilon}} S_{D}(z, \zeta)(1-f(z) \overline{f(\zeta)}) \rho(z) \overline{\rho(\zeta)} d \sigma(z) d \sigma(\zeta) \\
+\frac{1}{2} \int_{E}\left(1-|f|^{2}\right)|\rho|^{2} d \sigma \geqslant 0
\end{gathered}
$$

for all $\rho \in L^{\infty}(E)$, where $E$ is a Borel subset of $\partial D$ and

$$
(E \times E)_{\varepsilon}=\{(z, \zeta) \in E \times E:|z-\zeta|>\varepsilon\} .
$$

Once again, if $E$ is an arc on $\partial D$ and if $f \in C^{1}(E)$ with $|f|=1$ on $E$, then the second integral in (4.8) vanishes while the first integral is nonsingular. In this case, (4.8) becomes

$$
\int_{E} \int_{E} S_{D}(z, \zeta)(1-f(z) \overline{f(\zeta)}) \rho(z) \overline{\rho(\zeta)} d \sigma(z) d \sigma(\zeta) \geqslant 0, \quad \rho \in L^{\infty}(E) .
$$

The discrete version of this condition may be given by means of the kernel $K_{f}$ defined below. For $f$ as above, i.e. $f \in C^{1}(E)$ and $|f|=1$ on $E$, we define the kernel $K_{f}$ on $E \times E$ by

$$
K_{f}(z, \zeta)= \begin{cases}S_{D}(z, \zeta)(1-f(z) \overline{f(\zeta)}), & z \neq \zeta \\ \frac{1}{2 \pi i} \frac{d}{d s} \log f(\zeta), & z=\zeta\end{cases}
$$

where $s$ is the usual arc-length parameter on $\partial D$. Then (4.9) is equivalent to the positive-definiteness of the kernel $K_{f}$ on $E$. This extends the previously mentioned classical theorem of Loewner to multiply-connected plane domains.

ACKNOWLEDGEMENT. The first author acknowledges partial support from a Faculty Research Grant, FAS, University of Pittsburgh.

\section{REFERENCES}

1.N. Aronszajn, Theory of reproducing kernels, Trans. Amer. Math. Soc. 68 (1950), 337-404.

2. F. Beatrous, Jr., $H^{\infty}$ interpolation from a subset of the boundary, Pacific J. Math. 106 (1983), 23-31.

3. S. Bergman, The kernel function and conformal mapping, Math. Surveys, No. 5, Amer. Math. Soc., Providence, R. I., 1970.

4. J. Burbea, Pick's theorem with operator-valued holomorphic functions, Kōdai Math. J. 4 (1981), 495-507.

5. The Cauchy and the Szegö kernels on multiply connected regions, Rend. Circ. Mat. Palermo (2) 31 (1982), 105-118.

6. ___ Operator-valued Pick's conditions and holomorphicity, Pacific J. Math. 98 (1982), 295-311.

7. Inequalities for holomorphic functions of several complex variables, Trans. Amer. Math. Soc. 276 (1983), 247-266.

8. L_ Lower order positivity and holomorphic operators, J. Funct. Anal. 56 (1984), 1-14.

9. J. Burbea and P. Masani, Hilbert spaces of Hilbert space valued functions, Probability Theory on Vector Spaces. II, Lecture Notes in Math., vol. 828, Springer-Verlag, Berlin and New York, 1980, p. 1-20. 10. Banach and Hilbert spaces of vector-valued functions, their general theory and applications to holomorphy, Pitman Research Notes in Math., vol. 90, Pitman, London, 1984.

11. W. F. Donoghue, Monotone matrix functions and analytic continuation, Springer-Verlag, New York, 1974.

12. Reproducing kernel spaces and analytic continuation, Rocky Mountain J. Math. 10 (1980), $85-97$. 
13. P. L. Duren and A. L. Shields, Restrictions of $H^{p}$ functions to the diagonal of the polydisc, Duke Math. J. 42 (1975), 751-753.

14. C. H. FitzGerald, Quadratic inequalities and analytic continuation, J. Analyse Math. 31 (1977), $19-47$.

15.

, Conditions for a function on an arc to have a bounded analytic extension, The Johns Hopkins University, Tech. Report No. 303, 1978.

16. C. H. FitzGerald and R. A. Horn, On quadratic and bilinear forms in function theory, Proc. London Math. Soc. (3) 44 (1982), 554-576.

17. K. T. Hahn, Holomorphic functions with positive real part on complete circular domains (preprint).

18. C. Horowitz and D. Oberlin, Restrictions of $H^{p}$ functions on the diagonal of $U^{n}$, Indiana Math. J. 24 (1975), 767-772.

19. A. Korányi and L. Pukansky, Holomorphic functions with positive real part on polycylinders, Trans. Amer. Math. Soc. 108 (1963), 449-456.

20. A. Korányi and S. Vági, Singular integrals in homogeneous spaces and some problems of classical analysis, Ann. Scuola Norm. Sup. Pisa 25 (1971), 575-648.

21. K. Löwner, Über monotone Matrix funktionen, Math. Z. 38 (1934), 177-216.

22. B. Moulin and J. P. Rosay, Sur la restriction des functions plurisous-harmoniques a la diagonale $d u$ polydisque, Indiana Math. J. 26 (1977), 869-873.

23. R. Nevanlinna, Über beschränkte Funktionen die in gegeben Punkten vorgeschreibene Werte annehmen, Ann. Acad. Sci. Fenn. Ser. B 13 (1919).

24. G. Pick, Über die Beschränkungen analytischer Funktionen welche durch vorgegehene Funktionswerte bewirkt werden, Math. Ann. 77 (1916), 7-23.

25. M. Rosenblum and J. Rovnyak, Restrictions of analytic functions. I, Proc. Amer. Math. Soc. 48 (1975), 113-119.

26. W. Rudin, Function theory in polydiscs, Benjamin, New York, 1969.

27. Function theory in the unit ball of $\mathbf{C}^{n}$, Springer-Verlag, New York, 1980.

28. J. H. Shapiro, Mackey topologies, reproducing kernels, and diagonal maps on the Hardy and Bergman spaces, Duke Math. J. 43 (1976), 187-200.

29. E. M. Stein, Boundary hehavior of holomorphic functions of several complex variables, Princeton Univ. Press, Princeton, N. J., 1972.

30. S. E. Warschawski, On the differentiability at the boundary in conformal mapping, Proc. Amer. Math. Soc. 12 (1961), 614-620.

Department of Mathematics, University of Pittsburgh, Pittsburgh, Pennsylvania 15260 